\title{
Ground-state properties of one-dimensional matter and quantum dissociation of a Luttinger liquid
}

\author{
Eugene B. Kolomeisky*, Xiaoya Qi ${ }^{\dagger}$, and Michael Timmins \# \\ Department of Physics, University of Virginia, P. O. Box 400714, Charlottesville, VA 22904-4714
}

\begin{abstract}
Motivated by emerging experimental possibilities to confine atoms and molecules in quasi-onedimensional geometries, we analyze ground-state properties of strictly one-dimensional molecular matter comprised of identical particles of mass $m$. Such a class of systems can be described by an additive two-body potential whose functional form is common to all substances which only differ in the energy $\epsilon$ and range $l$ scales of the potential. With this choice De Boer's quantum theorem of corresponding states holds thus implying that ground-state properties expressed in appropriate reduced form are only determined by the dimensionless parameter $\lambda_{0}^{2} \sim \hbar^{2} / m l^{2} \epsilon$ measuring the strength of zero-point motion in the system. The presence of a minimum in the two-body interaction potential leads to a many-body bound state which is a Luttinger liquid stable for a not very large $\lambda_{0}$. As $\lambda_{0}$ increases, the asymmetry of the two-body potential causes quantum expansion, softening, and eventual evaporation of the Luttinger liquid into a gas phase. Selecting the pair interaction potential in the Morse form we analytically compute the properties of the Luttinger liquid and its range of existence. We find that as $\lambda_{0}$ increases, the system first undergoes a discontinuous evaporation transition into a diatomic gas followed by a continuous dissociation transition into a monoatomic gas. Two-body potentials of molecular systems can be successfully fitted into the Morse form thus allowing determination of the quantum parameter $\lambda_{0}$ and the state of matter of substance in question. In particular we find that spin-polarized isotopes of hydrogen and ${ }^{3} \mathrm{He}$ are monoatomic gases, ${ }^{4} \mathrm{He}$ is a diatomic gas, while molecular hydrogen and heavier substances are Luttinger liquids. We also investigate the effect of finite pressure on the properties of the liquid and monoatomic gas phases. In particular we estimate a pressure at which molecular hydrogen undergoes an inverse Peierls transition into a metallic state which is a one-dimensional analog of the transition predicted by Wigner and Huntington in 1935.
\end{abstract}

PACS numbers: 68.65.-k, 61.46.+w, 71.10.Pm, 05.30.-d

\section{INTRODUCTION}

Prediction of the ground-state properties of a condensed many-body system of identical particles starting from microscopic two-body interactions is only meaningful if the outer electronic shells of underlying atoms or molecules in bound phase are not very different from their free state counterparts [1]. If the two-particle potential has a functional form common to a family of substances (for example, of the Lennard-Jones type), then the properties of all the members of the family can be related. This conclusion pioneered by De Boer and collaborators, commonly referred as the quantum theorem of corresponding states, was originally applied to predict the properties of ${ }^{3} \mathrm{He}[2]$ before it had become experimentally available. Later Anderson and Palmer [3] and Clark and Chao [4] used the same approach to estimate the properties of zero-temperature nuclear and neutron-star matter from those of laboratory substances.

The goal of this work is to conduct a similar program in a strictly one-dimensional case. There are several reasons, both of fundamental and practical nature, why it is important to understand this problem.

First, it is well-known that for ordinary substances zero-point motion is of crucial importance only for the lightest elements such as helium isotopes as well as spinpolarized isotopes of hydrogen. The ground state of all heavier elements (and molecular hydrogen) is crystalline and to large extent classical. The one-dimensional case is qualitatively different: regardless of the particle mass zero-point motion destroys the long-range crystalline order - a situation that is closely analogous to the destruction of the long-range order by thermal fluctuations in classical two-dimensional systems of continuous symmetry [5]. As a result, the only possible many-body bound state in one dimension is a harmonic or Luttinger liquid a uniform density condensed phase with algebraically decaying density correlations [6]. This decay, characterized by a nonuniversal exponent, is slower than the exponential fall-off of density correlations in conventional fluids. As the degree of zero-point motion increases, the manybody bound state can disappear through a transition that has no analog in the three-dimensional world: since the Luttinger liquid phase is more correlated than conventional fluids and less correlated than standard crystals its dissociation combines qualitative features of both laboratory melting and evaporation at once.

Second, in one dimension the difference between fermions and bosons is not very significant as we cannot go from one configuration to another with exchanged particles without bringing the particles in contact at some intermediate step. Then short-distance repulsion among bosons has the same effect on density correlations as the Pauli principle for fermions. On the other hand, zero- 
temperature properties of three-dimensional matter with an interaction pair potential of the Lennard-Jones form are sensitive to the statistics of the underlying particles $[7]$.

Since experimental discovery of carbon nanotubes in 1991 [8] studying the properties of one-dimensional systems became especially important. In addition to their unique transport, mechanical and chemical properties [9], bundles of carbon nanotubes can play a role of onedimensional hosts for foreign atoms that can find themselves bound in the interstitial channels or inside the tubes [10]. One of the interesting potential applications of these systems includes storage devices for molecular hydrogen in fuel cells [11].

Recently the quasi-one-dimensional regime has been also realized for Bose-condensates of alkali vapors both for repulsive [12] and attractive interactions [13]. These systems which are relevant for atom interferometry [14] have an additional flexibility as the strength and sign of two-body interactions can be magnetically tuned.

In both of these experimental examples the basic model is a zero-temperature one-dimensional many-body system of fermions or bosons with pairwise interactions. There were several attempts in the past to study this problem:

(i) Diffusion Monte Carlo studies predicted that at zero temperature both one-dimensional ${ }^{4} \mathrm{He}[15,16]$ and molecular hydrogen [17] form weakly-bound liquids.

(ii) These conclusions were supported by variational studies based on the Jastrow-Feenberg wave function [18] where additionally it was argued that in one dimension the many-body bound state exists only for those systems which have a dimer, i. e. a two-body bound state. One of the findings common to Refs. [16-18] is the prediction of a high-density liquid-solid phase transition in which a standing density wave sets in. We note however that the existence of such a one-dimensional solid contradicts the quantum version of the Mermin-Wagner-Hohenberg theorem [5].

(iii) A direct variational treatment based on a Gaussian wave function was performed in Ref. [19] where it was assumed that the particles form a one-dimensional chain with the Morse potential interaction [20] between nearest neighbors. Although it was shown that the chain remained stable for not very strong quantum fluctuations, the accuracy of the method, the nature of the condensed phase, the role of dimerization, and the implications for real systems were not addressed.

In this paper the problem of the ground-state properties of a one-dimensional many-body system is reexamined for the case when the two-body interparticle interaction can be approximated by the Morse potential [20]. Since it involves three parameters, the Morse potential is more flexible in describing real systems as compared to the two-parameter Lennard-Jones potential. At the same time the quantum theorem of the corresponding states [2] still holds in this case. In addition the problem of the Morse dimer is exactly solvable [20]; below we will also show that analytical progress is possible in the many-body case, and the accuracy of our results can be assessed.

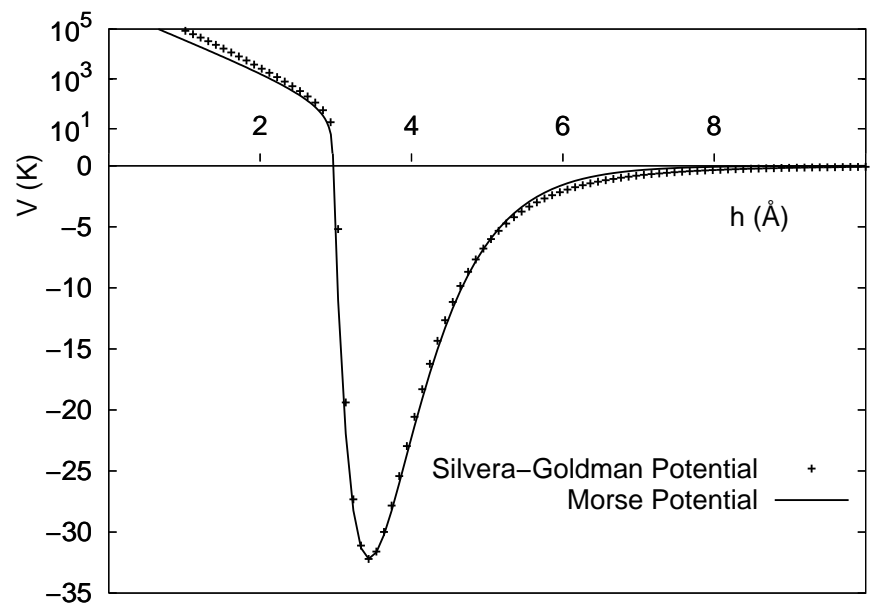

FIG. 1. The Silvera-Goldman interaction potential between two hydrogen molecules (crosses) and its approximation by the Morse potential (solid curve). To demonstrate the strength of the overlap repulsion, logarithmic scale is used on the positive energy axis. The details of the fit are explained in the text.

The organization of the paper is as follows. In Section II we set up the problem in general terms with the quantum theorem of the corresponding states [2] as a guide for possible outcomes. The parameters of the Luttinger liquid are computed in Sections III-V. In Section III we describe general properties of the Luttinger liquid and outline the main idea of the calculation. Zero-pressure analysis is carried out in Section IV. The main tool here is a combination of variational and renormalization-group treatments. As a by-product we also solve the problem of quantum Brownian motion in the Morse potential, and show that it exhibits a localization-delocalization transition. Finite-pressure variational analysis is conducted in Section V. Section VI is dedicated to the discussion and applications of our results to various molecular substances. In particular we estimate a pressure at which one-dimensional molecular hydrogen undergoes a transition into a metallic state.

\section{FORMULATION OF THE PROBLEM}

Our starting point is the Euclidian action for $N$ identical fermions or bosons of mass $m$ with pairwise interactions

$$
S=\int d \tau\left[\frac{m}{2} \sum_{i=1}^{N}\left(\frac{d x_{i}}{d \tau}\right)^{2}+\sum_{i<j}^{N} V\left(x_{i}-x_{j}\right)\right],
$$


where $\tau$ is the imaginary time variable, $x_{i}$ are particle positions, and $V(h)$ is the pair interaction potential. The properties of the function $V(h)$ can be summarized as follows. At large separation $h$ interparticle interaction is dominated by weak rapidly decaying Van der Waals attraction, while at short distances there is a very strong overlap repulsion [1]. As a result, the pair potential $V(h)$ has an asymmetric minimum at some intermediate $h$. As a typical example Fig. 1 shows the semi-empirical SilveraGoldman potential [21] between two $H_{2}$ molecules which is extensively used in computations of the properties of molecular hydrogen.

Assume that the pair potential has the form

$$
V(h)=\epsilon U\left[\left(h-H_{0}\right) / l\right],
$$

where $\epsilon$ is the energy scale of the potential, $l$ is the potential range, $H_{0}$ is a length scale, and $U(y)$ is a function common to a family of substances. Introducing dimensionless position and time variables, $q_{i}=x_{i} / l, t=\epsilon \tau / \hbar$, transforms the reduced action $S / \hbar$ into

$$
\frac{S}{\hbar}=\int d t\left[\frac{m \epsilon l^{2}}{2 \hbar^{2}} \sum_{i=1}^{N}\left(\frac{d q_{i}}{d t}\right)^{2}+\sum_{i<j}^{N} U\left(q_{i}-q_{j}-Q_{0}\right)\right],
$$

where $Q_{0}=H_{0} / l$. The quantum theorem of the corresponding states [2] then directly follows from representation (3): the energy per particle $E^{*}$ measured in units of $\epsilon$ is only determined by the dimensionless parameter

$$
\lambda_{0}=\frac{\hbar}{\pi l(2 m \epsilon)^{1 / 2}},
$$

the form of the function $U$ in (2), and the particle statistics:

$$
E^{*}=E^{*}\left(\lambda_{0}, \text { statistics }\right)
$$

Similar statements can be made about the reduced relative equilibrium length per particle $Q-Q_{0}(Q$ is the one-dimensional version of volume per particle measured in units of $l$ ), and other quantities of interest. Apart from numerical factors (introduced for convenience), the quantum parameter $\lambda_{0}$ (4) is identical to De Boer's number [2]: its square is proportional to the ratio of the zeropoint energy of particle of mass $m$ localized within range $l$ to the typical potential energy $\epsilon$. Therefore as $\lambda_{0}$ increases away from its classical value $\lambda_{0}=0$, the strength of zero-point motion increases.

In the presence of several competing phases the function $E^{*}$ in (5) (and other properties) will have several branches; the branch with lowest $E^{*}$ singles out the ground-state of the system. When two different branches cross, the ground-state changes via a first-order phase transition. Each branch of $E^{*}\left(\lambda_{0}\right)$ is an analytical function of its argument except possibly at isolated points where critical phenomena take place. One obvious branch of $E^{*}$ corresponds to a monoatomic gas which must become the ground state of the system at sufficiently large $\lambda_{0}$. Then all the particles are infinitely far apart from each other, and thus $E_{\text {mono }}^{*}\left(\lambda_{0}\right)=0$ which we select to be the reference point for the energy.

In what follows we select the pair interaction potential in the Morse form [20]:

$$
\begin{aligned}
V(h) & =-A e^{-h / l}+B e^{-2 h / l} \\
& \equiv \epsilon\left[-2 e^{-\left(h-H_{0}\right) / l}+e^{-2\left(h-H_{0}\right) / l}\right],
\end{aligned}
$$

where $A$ and $B$ are the amplitudes of the attractive and repulsive parts of the potential, respectively, $H_{0}=$ $l \ln (2 B / A)$ is the location of the minimum of (6), while $\epsilon=V\left(H_{0}\right)=A^{2} / 4 B$ is the depth of the potential well. The second representation of (6) shows explicitly that the Morse potential conforms to the general form (2). It is physically reasonable to require that the zero of $(6)$ is located at positive $h$ which implies $B>A$.

Similar to the applications of the Lennard-Jones potential to laboratory molecular systems [1], Eq.(6) should not be taken too literally as really describing two-particle interactions. The only reason behind our choice (6) is the possibility of analytic progress.

For two particles interacting according to (6) the ground-state energy is exactly known to be $E_{2}=-\epsilon[1-$ $\left.\hbar / 2 l(m \epsilon)^{1 / 2}\right]^{2}[20]$. This implies that in the many-body case one of the possible phases of the system is a diatomic gas (a collection of infinitely far separated dimers) with the reduced energy function

$$
E_{\text {dimer }}^{*}\left(\lambda_{0}\right)=-\frac{1}{2}\left(1-\frac{\pi \lambda_{0}}{\sqrt{2}}\right)^{2},
$$

valid for $\lambda_{0} \leq \lambda_{02}=\sqrt{2} / \pi$; the factor of $1 / 2$ accounts for two particles in the dimer. As $\lambda_{0}$ approaches $\lambda_{02}$ from below, the dimer size diverges, and at $\lambda_{0}=\lambda_{02}$ a second-order dissociation transition into the monoatomic gas discussed earlier takes place. The asymmetry of the interaction potential is responsible for the disappearance of the two-body bound state for sufficiently strong zeropoint motion.

The diatomic gas might be the ground-state of the system for intermediate $\lambda_{0}$ but for sufficiently small $\lambda_{0}$ a condensed phase must have the lowest energy. For molecular systems in general [1] pair interactions decay rapidly with interparticle separation. As a result, the physics of the condensed phase is dominated by nearest-neighbor interactions. Therefore in what follows in describing the one-dimensional condensed phase we restrict ourselves to nearest-neighbor interactions. Corrections coming from ignoring distant neighbors will be marginally small provided the interaction range $l$ is substantially smaller than average interparticle spacing (bond length).

In the classical limit, $\lambda_{0}=0$, the ground-state of the system is a one-dimensional crystal of particles with lattice spacing $H_{0}$ given by the minimum of the two-body Morse potential (6). Indeed, the energy per particle for 
the crystal, $-\epsilon$, is twice as negative as that for the diatomic gas.

A condensed phase is also the ground-state of the system for a not very large $\lambda_{0}$. The quantitative theory of the properties of this phase is developed below.

\section{LUTTINGER LIQUID PHASE AND ITS PROPERTIES}

When zero-point motion is present, the asymmetry of the pair interaction potential about its minimum causes quantum expansion. As a result the average bond length $H$ in the condensed state exceeds its classical, $\lambda_{0}=0$, counterpart $H_{0}$. The low-energy dynamics of the system is described by the harmonic action [6]

$$
S_{h a r m}=\frac{\rho}{2} \int d x d \tau\left[\left(\frac{\partial u}{\partial \tau}\right)^{2}+c^{2}\left(\frac{\partial u}{\partial x}\right)^{2}\right],
$$

where $u(x, \tau)$ is the particle displacement field, $\rho=m / H$ is the mass density, and $c$ is the sound velocity [1]

$$
c^{2}=\frac{H^{2}}{m} \frac{\partial^{2} E(h=H)}{\partial h^{2}},
$$

where $E(h)$ is the ground-state energy per particle as a function of (one-dimensional) volume per particle $h$, and the derivative is evaluated at the equilibrium interparticle spacing $H$. The function $E(h)$ can be also viewed as an effective pair interaction renormalized by zero-point motion away from its classical form (6).

The Feynman path integral formulation of quantum mechanics [22] allows us to view the action (8) as a Hamiltonian for a classical two-dimensional crystal of line objects (world lines of underlying particles) running in the imaginary time direction. In this correspondence zero-point motion plays a role of thermal fluctuations. But this is exactly the context of applicability of the Mermin-Wagner-Hohenberg theorem [5]: if $n(x, \tau)$ is the instantaneous number density, then long-wavelength lowenergy quantum fluctuations captured by the action (8) destroy long-range positional order of the particles. The only allowed many-body bound state is a uniform density phase, $<n(x, \tau)>=H^{-1}(<>$ stands for the expectation value), with algebraic decay of density correlations $[6]$ :

$$
<n(x, \tau) n(0,0)>-H^{-2} \propto \frac{\cos (2 \pi x / H)}{\left(x^{2}+c^{2} \tau^{2}\right)^{g}},
$$

where the exponent $g$ is given by

$$
g=\frac{\pi \hbar}{\rho c H^{2}}
$$

The large distance/time behavior of the density-density correlation function (10) is the hallmark of the Luttinger liquid. In order to compute the range of existence of the Luttinger liquid phase, its bond length $H$, sound velocity $c$, and correlation exponent $g$ we need to go beyond harmonic approximation.

Our calculation relies on the assumption underlying the harmonic description (8) (and to be verified later) that as long as the Luttinger liquid is stable, the ratio of the typical fluctuation of the bond length to the bond length itself remains small despite individually both these quantities are increasing functions of $\lambda_{0}(4)$. Then every bond of the system can be viewed as a quantummechanical degree of freedom subject to the external potential $V(h)$ and placed in contact with a bath of harmonic oscillators (8) corresponding to the rest of the system. The single bond dynamics is thus given by the action:

$$
S_{\text {bond }}=\frac{\rho}{2} \int_{\text {bath }} d x d \tau\left[\left(\frac{\partial u}{\partial \tau}\right)^{2}+c^{2}\left(\frac{\partial u}{\partial x}\right)^{2}\right]+\int d \tau V(h)
$$

The first integral is over all positions and times except for a small region near $x=0$ where the bond in question is located. This separates the system into two pieces, so that at all times the displacement field $u$ is discontinuous: $u(x=+0, \tau)-u(x=-0, \tau)=h(\tau)-H_{0}$. The coupling between the segments of the system joined at the bond is given by the full pair potential $V(h)$, i. e. it goes beyond harmonic approximation.

The action (12) has been previously introduced in Ref. [23] to describe tunneling-assisted fracture of a stretched one-dimensional chain. More generally this type of action corresponds to the Caldeira-Leggett model of coupling between a quantum-mechanical degree of freedom and an environment modeled by a reservoir of harmonic oscillators [24]. Since the last interaction term in (12) is restricted to a single spatial point, the bath degrees of freedom can be integrated out away from the bond with the result [23]:

$$
S=\frac{\rho c}{4} \int_{-\omega_{D}}^{\omega_{D}} \frac{d \omega}{2 \pi}|\omega||h(\omega)|^{2}+\int d \tau V(h),
$$

where the subscript is dropped for brevity and the Fourier transform of the bond length field $h(\omega)=$ $\int h(\tau) \exp (-\omega \tau) d \tau$ has been introduced [25]. The frequency cutoff $\omega_{D}$ setting the limits of integration in the first kinetic term of (13) is the one-dimensional Debye frequency $\omega_{D}=\pi c / H$ - the vibrational spectrum of the system is approximated by the Debye model.

The unusual $|\omega|$ dependence of the kinetic term of the action (13) is due to the many-body nature of the bond dynamics, and can be understood heuristically by noticing that if the bond length oscillates with frequency $\omega$, then during one oscillation period $2 \pi /|\omega|$ this disturbance propagates in both directions away from the bond a distance of order $c /|\omega|$. Therefore the standard kinetic energy density, proportional to $\rho \omega^{2}$ should be multiplied by 
the size of the region $c /|\omega|$ affected by the motion thus reproducing the $\rho c|\omega|$ term of (13).

The action (13) allows us in principle to compute how the bath degrees of freedom renormalize the properties of a given bond. The nonanalytic $|\omega|$ dependence in (13) guarantees that the bond cannot renormalize the bath oscillators (whose properties are accumulated in the $\rho c$ combination). This observation combined with the fact that all the bounds of the Luttinger liquid are equivalent provides us with a prescription on how to use (13) to solve the problem we are interested in:

The parameters $\rho$ and $c$ of (13) should be selected as initially unknown but fully renormalized properties of the Luttinger liquid. The reservoir degrees of freedom will renormalize the microscopic pair interaction $V(h)$ into a form which we will require to be identical (in the harmonic limit) to the rest of the chain. This will determine the parameters of the Luttinger liquid and guarantee that the treatment is insensitive to the choice of the bond.

Since the action (13) describes the dynamics of an arbitrary single bond of the system, it can be directly used to compute the energy per particle of the original manybody problem.

\section{ZERO-PRESSURE ANALYSIS}

For a quantitative analysis we use Feynman's [26] variational principle for the ground-state energy:

$$
E \leq E_{1}=E_{0}+(T / \hbar)<S-S_{0}>_{0}
$$

where $T$ is the temperature, and $\hbar / T$ has a meaning of the system size in the $\tau$ direction; the $T=0$ limit will be taken at the end. The notation $<>_{0}$ denotes an expectation value computed using an arbitrary reference action $S_{0}$, and $E_{0}$ is the ground-state energy corresponding to $S_{0}$.

This method has been remarkably successful in analyzing the roughening phase transition [27], multilayer adsorption phenomena [28], wetting transitions [29], the problem of quantum Brownian motion in a periodic potential [30], the Coulomb blockade problem [31], and a variety of problems of quantum mechanics and quantum field theory [32].

It is physically reasonable to select the trial action $S_{0}$ in a Gaussian form similar to that in [28], [29], and [31]:

$$
\left.S_{0}=\frac{\rho c}{4} \int_{-\omega_{D}}^{\omega_{D}} \frac{d \omega}{2 \pi}|\omega||h(\omega)|^{2}+\frac{K}{2} \int d \tau(h-H)^{2}\right],
$$

where two variational parameters which include the familiar bond length $H$ and a new parameter $K$ (controlling the extent of fluctuations about $H$ ) are selected to minimize $E_{1}$ in (14). The stiffness parameter $K$ has a meaning of the curvature of the effective pair potential evaluated at its minimum $H$, and appearing in (9), $K=\partial_{h}^{2} E(h=H)$.

Introducing $f=h-H$, the deviation of the bond length away from its equilibrium value $H$, the reduced root-mean-square (rms) fluctuation, $f^{*}=\left(<f^{2}>_{0}\right.$ $\left./ l^{2}\right)^{1 / 2}$, can be computed with the help of (15) as

$$
f^{*}=(2 \lambda)^{1 / 2} \ln ^{1 / 2}\left(1+\gamma^{-1}\right),
$$

where

$$
\lambda=\frac{\hbar}{\pi \rho c l^{2}}
$$

is the dimensionless parameter quantifying the strength of zero-point motion in the Luttinger liquid, and

$$
\gamma=\frac{2 K}{\omega_{D} \rho c}
$$

is the dimensionless counterpart of $K$. For the Morse pair interaction (6) the classical sound velocity is $c_{0}=$ $\left(H_{0} / l\right)(2 \epsilon / m)^{1 / 2}$. It is then straightforward to verify that in the classical limit, $\hbar \rightarrow 0$, the quantum parameter $\lambda$ (17) reduces to De Boer's number $\lambda_{0}(4)$.

In the Luttinger liquid the strength of zero-point motion is characterized by the correlation exponent $g$ (11) which is related to $\lambda(17)$ by

$$
g=\lambda \pi^{2} / Q^{2}
$$

where $Q=H / l$ is the reduced bond length. This relationship demonstrates that if the interaction range $l$ is known, then measuring the density-density correlation function (10) will allow us to compute $\lambda$, and thus the rms fluctuation of the bond length (16).

Using (15), (16), and (6) the reduced upper bound $E^{*}=E_{1} / \epsilon$ entering (14) can be computed as

$$
\begin{aligned}
E^{*}(\gamma, Q) & =v^{-1} \ln (1+\gamma)-2 e^{Q_{0}-Q}\left(1+\gamma^{-1}\right)^{\lambda} \\
& +e^{2\left(Q_{0}-Q\right)}\left(1+\gamma^{-1}\right)^{4 \lambda}
\end{aligned}
$$

where

$$
v=\frac{2 \pi \epsilon}{\hbar \omega_{D}}
$$

is related to the reduced Debye temperature as $\theta^{*}=$ $\hbar \omega_{D} / \epsilon=2 \pi / v$.

The expression for $E^{*}$ should be minimized with respect to $\gamma$ and $Q$, and in case of multiple solutions the one minimizing (20) must be selected.

Minimizing $E^{*}$ with respect to $Q$ we arrive at the expression for the reduced bond length which accounts for quantum expansion

$$
Q=Q_{0}+3 \lambda \ln \left(1+\gamma^{-1}\right)
$$


Substituting this back into (20) the expression for the reduced energy $E^{*}$ can be written as

$$
E^{*}(\gamma)=v^{-1} \ln (1+\gamma)-\left(1+\gamma^{-1}\right)^{-2 \lambda},
$$

Minimizing (23) with respect to $\gamma$ we find

$$
\gamma=2 v \lambda\left(1+\gamma^{-1}\right)^{-2 \lambda}
$$

\section{A. Approximate solution: $\lambda=\lambda_{0}$}

First we look at a simplified version of the original problem when only one bond of the system is subject to the Morse potential (6) while the rest of the chain is harmonic. This situation is described by the action

$$
\begin{aligned}
S & =\frac{\rho_{0} c_{0}}{4} \int_{-\omega_{D 0}}^{\omega_{D 0}} \frac{d \omega}{2 \pi}|\omega||h(\omega)|^{2} \\
& +\int d \tau\left(-A e^{-h / l}+B e^{-2 h / l}\right),
\end{aligned}
$$

where $\rho_{0}=m / H_{0}, c_{0}=\left(H_{0} / l\right)(2 \epsilon / m)^{1 / 2}$, and $\omega_{D 0}=$ $\pi c_{0} / H_{0}$ assume their classical values. The parameters $A$ and $B$ are selected so that in the harmonic approximation the Morse bond is identical to the rest of the chain.

If the imaginary time coordinate $\tau$ is viewed as a fictitious space variable then (25) can be recognized as an effective Hamiltonian defining a classical statistical mechanics problem [22]. If the bond field $h$ is identified with an interface height and zero-point motion with thermal fluctuations, then this problem is a one-dimensional analog of the critical wetting problem [29], [33].

For finite $\lambda_{0}$ zero-point motion softens and lengthens the Morse bond while leaving the harmonic part of the chain intact. This is described by Eqs.(22)-(24) with $\lambda=$ $\lambda_{0}$ and $v=v_{0}=1 /\left(\pi \lambda_{0}\right)$. Specifically, Eqs.(23) and (24) turn into:

$$
\begin{gathered}
E^{*}(\gamma)=\pi\left[\lambda_{0} \ln (1+\gamma)-\frac{\gamma}{2}\right] \\
\gamma=\frac{2}{\pi}\left(1+\gamma^{-1}\right)^{-2 \lambda_{0}}
\end{gathered}
$$

First we note that $\gamma=0$ is always a solution to (27) with $E^{*}=0$ which describes two segments of the chain infinitely far apart from each other. As $\lambda_{0}$ increases away from the classical value $\lambda_{0}=0$, the parameter $\gamma$ in (27) monotonically decreases from $\gamma=2 / \pi$ vanishing at $\lambda_{0}=$ $1 / 2$. For $\lambda_{0}>1 / 2$ only $\gamma=0$ solves Eq.(27). When $\lambda_{0}$ approaches the critical value of $1 / 2$ from below we have

$$
\gamma \simeq e^{-\frac{\ln (\pi / 2)}{1-2 \lambda_{0}}}
$$

Correspondingly, the reduced energy (26) monotonically increases with $\lambda_{0}$ : in the classical limit, $\lambda_{0} \rightarrow 0$, it rises linearly with $\lambda_{0}$ according to $E^{*}=-1+\lambda_{0}[\pi \ln (1+2 / \pi)+$ $2 \ln (1+\pi / 2)] \simeq-1+3.4361 \lambda_{0}$. Since $\lambda_{0} \sim 1 / \mathrm{m}^{1 / 2}$, Eq. (4), the energy $E^{*}$ is not analytic as $1 / m \rightarrow 0$, which is the relevant quantity [3]. This is expected because the crystal $\left(\lambda_{0}=0\right)$ is qualitatively different from the Luttinger liquid (finite $\lambda_{0}$ ). Upon approaching $\lambda_{0}=1 / 2$ from below the reduced energy vanishes as

$$
E^{*} \simeq-\frac{\pi}{2}\left(1-2 \lambda_{0}\right) e^{-\frac{\ln (\pi / 2)}{1-2 \lambda_{0}}}
$$

As $\lambda_{0}$ approaches $1 / 2$ the bond length diverges and for $\lambda_{0} \geq 1 / 2$ the two segments of the chain are infinitely far apart from each other.

The behavior of the reduced bond length and its rms fluctuation just below the phase transition can be found by combining (16) and (22) with (28):

$$
Q \simeq \frac{3 \ln (\pi / 2)}{2\left(1-2 \lambda_{0}\right)}, \quad f^{*} \simeq \frac{\ln ^{1 / 2}(\pi / 2)}{\left(1-2 \lambda_{0}\right)^{1 / 2}}
$$

We note that although both of these quantities diverge upon approaching the phase transition, the relative fluctuation, $f^{*} / Q$, vanishes. Thus fluctuating segments of the chain never overlap and our treatment is consistent.

The essential singularities (28) and (29) at $\lambda_{0}=1 / 2$ as well as the divergences (30) parallel those found in the context of wetting transitions [29].

The most valuable feature of the variational approach is its nonperturbative nature. The accuracy of variational predictions depends on how close is the variational guess to the physical reality. For the problem defined by the action (25) the accuracy of our approach can be assessed and the special role played by $\lambda_{0}=1 / 2$ can be re-established by using a renormalization-group method.

\section{B. Perturbative renormalization-group treatment:

$$
\lambda=\lambda_{0}
$$

Following the argument of Brézin, Halperin, and Leibler originally given in the classical context of wetting transitions [33] we treat the Morse potential term in (25) as a perturbation. Then the lowest-order renormalization-group equations have the form:

$$
\frac{d \ln A}{d \ln \left(\omega_{D 0} \zeta\right)}=\left(\lambda_{0}+1\right), \quad \frac{d \ln B}{d \ln \left(\omega_{D 0} \zeta\right)}=\left(4 \lambda_{0}+1\right),
$$

where $\zeta$ is the running scale in the $\tau$ direction, and the equations describe how the Morse parameters $A$ and $B$ renormalize upon (i) successive integration out of highfrequency modes (first terms) followed by (ii) scaling transformation which restores the cutoff to its original value (second terms). Instead of following separate evolution of the coefficients $A$ and $B$, it is more appropriate to look at the depth of the Morse well (6) $\epsilon=A^{2} / 4 B$ which is also proportional to the potential curvature at 
its minimum. For its dimensionless counterpart $v(21)$ Eqs.(31) imply

$$
\frac{d v}{d \ln \left(\omega_{D 0} \zeta\right)}=\left(1-2 \lambda_{0}\right) v
$$

The flow diagram corresponding to (32) is sketched in Fig. 2 where we also show the locus of initial conditions $v_{0}=1 / \pi \lambda_{0}$ of the model (25).

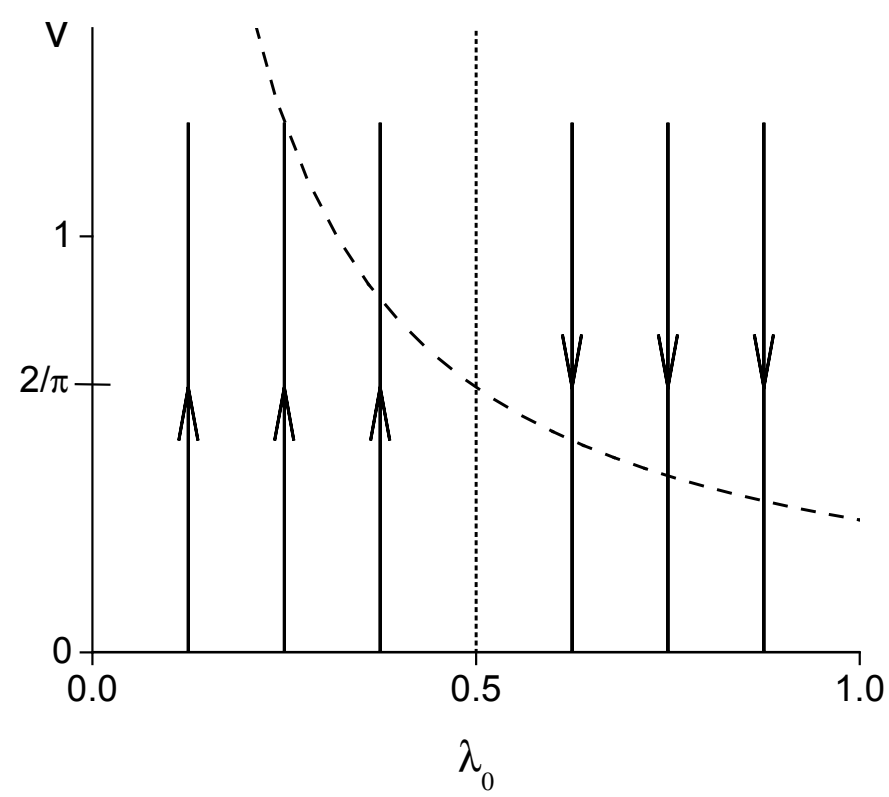

FIG. 2. The flow diagram of the model (25). The arrows indicate the direction of the flow and the dashed line $v_{0}=1 / \pi \lambda_{0}$ is the locus of initial conditions. The stable part of the $v=0$, $\lambda_{0}>1 / 2$ fixed line corresponds to two segments of the chain infinitely far away from each other. For $\lambda_{0}<1 / 2$ the parameter $v$ is relevant and the Morse bond joins the segments together.

First we note that verticality of the flow lines is a rigorous property of the model (25) as the $\rho_{0} c_{0}$ combination (and thus $\lambda_{0}$ ) does not renormalize to any order in $v$. There are clearly two regimes separated by a phase transition at the critical value $\lambda_{0}=1 / 2$. For $\lambda_{0}>1 / 2$ the parameter $v$ flows to zero which means that the chain is broken into two infinitely separated segments. On the other hand, for $\lambda_{0}<1 / 2$ the parameter $v$ grows under renormalization eventually leaving the perturbative regime $v \ll 1$. This means that the Morse bond holds both segments of the chain together. Upon approaching the critical value $\lambda_{0}=1 / 2$ from below there is a divergent time scale $\xi$ (analog of a correlation length in standard critical phenomena) which can be found from the condition $v(\xi) \simeq 1$ :

$$
\xi \simeq \omega_{D 0}^{-1} e^{\frac{\ln \left(1 / v_{0}\right)}{1-2 \lambda_{0}}}
$$

The one-dimensional nature of the problem then implies that the reduced curvature $\gamma$ (18) of the renor- malized potential vanishes as $\xi^{-1}$. Similarly the critical behavior of the reduced bond length $Q$ can be found as $Q \simeq \ln [B(\xi) / A(\xi)] \simeq(3 / 2) \ln \left(\omega_{D 0} \xi\right)$, while its rms fluctuation is $f^{*} \simeq \ln ^{1 / 2}\left(\omega_{D 0} \xi\right)$. With $\xi$ given by (33) and logarithmic accuracy these results coincide with their variational counterparts, Eq.(28) and (30). If we set the bare parameter $v_{0}$ in (33) at $2 / \pi$ (the crossing of the locus of initial conditions $v_{0}=1 / \pi \lambda_{0}$ and the $\lambda_{0}=1 / 2$ line) the renormalization-group results would become identical to those of the variational approach.

From perturbative renormalization-group treatment alone we would not be able to make reliable statements about the phase transition at $\lambda_{0}=1 / 2$ as the analysis is valid for $v_{0} \ll 1$ while for the problem in question (25) one has $v_{0}=2 / \pi \simeq 0.64<1$ - it is on the border of applicability of perturbative theory. However combining the above results with the nonperturbatve variational analysis makes a strong case. Since the latter produces the same answers in the region of parameters where renormalization-group results are less certain, we argue that the variational solution of (25) is very accurate in the range of $\lambda_{0}$ between the classical limit $\lambda_{0}=0$ and the dissociation transition $\lambda_{0}=1 / 2$ which is described exactly.

As a side observation we note that the problem (25) is most likely to be related to that of quantum Brownian motion of a particle in a periodic potential [30]. Superficially the only similarity between the two is that (25) can be also viewed as describing quantum Brownian motion in the Morse potential which has no periodicity.

The similarity between the problems becomes noticeable if one inspects their treatments. A comparison shows that our expression for variational energy (23) is identical to that of the periodic version of the problem with the amplitude of the periodic potential proportional to our parameter $v(21)$ and our $\lambda=\lambda_{0}$ corresponding to $1 / 2 \alpha$ of Fisher and Zwerger [30]. With this identification renormalization-group equation (32) coincides with its periodic counterpart [30]. Both problems have delocalization transitions of the same universality class driven by zero-point motion.

We remind the reader that the action (25) is an approximation to the original problem of the ground-state properties of the Luttinger liquid - only one bond is subject to the Morse potential while the rest of the system is purely harmonic. This chain is obviously stiffer than the original system - a smaller level of zero-point motion will be necessary to cause dissociation of the Luttinger liquid. Therefore the results derived for the model (25) imply that zero-pressure Luttinger liquid phase cannot exist for $\lambda_{0} \geq 1 / 2$.

We also note that the critical value $\lambda_{0}=1 / 2$ is larger than the dimer dissociation threshold $\lambda_{02}=\sqrt{2} / \pi \simeq$ 0.4502 . This can be understood qualitatively by noticing that the dynamics of the Morse bond joining two 
half-infinite harmonic segments is more inertial (and thus more classical) than that of the Morse dimer. Therefore with the same underlying particles a weaker level of quantum fluctuations (i. e. smaller $\lambda_{0}$ ) suffices to break the dimer.

\section{Accurate solution}

In order to compute the properties of the Luttinger liquid more accurately we have to impose the condition that all the bonds of the chain are equivalent. Then using definitions of De Boer's number $\lambda_{0}(4)$ and its Luttinger liquid counterpart $\lambda(17)$ the parameter $v$ (21) can be calculated as $v=\lambda / \pi \lambda_{0}^{2}$; for $\lambda=\lambda_{0}$ it reduces to $v=$ $1 / \pi \lambda_{0}$ previously used in approximate treatment of the problem. Similarly the reduced curvature of the effective pair potential (18) can be computed with the help of Eq.(9) with the conclusion that $\gamma=2 / \pi$. This is exactly what was previously found in the approximate analysis in the classical limit, $\lambda_{0}=0$, when indeed all the bonds of the chain are equivalent.

Substituting $v=\lambda / \pi \lambda_{0}^{2}$, and $\gamma=2 / \pi$ in Eq.(20) we arrive at the expression for the reduced energy as a function of dimensionless "volume" per particle $Q$ :

$$
\begin{aligned}
E^{*}(Q) & =\left(\pi \lambda_{0}^{2} / \lambda\right) \ln (1+2 / \pi)-2 e^{Q_{0}-Q+\lambda \ln (1+\pi / 2)} \\
& +e^{2\left(Q_{0}-Q\right)+4 \lambda \ln (1+\pi / 2)}
\end{aligned}
$$

Similarly Eqs.(16), (22)-(24) transform into

$$
\begin{gathered}
f^{*}=(2 \lambda)^{1 / 2} \ln ^{1 / 2}(1+\pi / 2) \\
Q=Q_{0}+3 \lambda \ln (1+\pi / 2) \\
E_{L L}^{*}=e^{-2 \lambda \ln (1+\pi / 2)}[\pi \lambda \ln (1+2 / \pi)-1] \\
\lambda_{0}=\lambda e^{-\lambda \ln (1+\pi / 2)}
\end{gathered}
$$

Eqs.(34)-(37) are the main results of this Section. We note that in view of Eqs.(10) and (19) Eqs.(35)-(37) give the dependence of the reduced rms bond length fluctuation $f^{*}$, bond length $Q$, and energy per particle of the Luttinger liquid $E_{L L}^{*}$ on the experimentally measurable quantum parameter $\lambda$.

The dependence of De Boer's number $\lambda_{0}$ on $\lambda$ is given by Eq.(38); we are interested in the inverse dependence, $\lambda\left(\lambda_{0}\right)$.

The right-hand side of (38) has a maximum at $\lambda_{s}=$ $\ln ^{-1}(1+\pi / 2) \simeq 1.0591$ of magnitude $\lambda_{0 s}=e^{-1} \ln ^{-1}(1+$ $\pi / 2) \simeq 0.3896$. For $\lambda_{0}<\lambda_{0 s}$ Eq.(38) has two roots for $\lambda$ but only the smaller one is physical. For $\lambda_{0}=\lambda_{0 s}$ these two roots coincide, and for $\lambda_{0}>\lambda_{0 s}$ Eq.(38) has no solutions - Luttinger liquid phase is no longer stable. We note that the Luttinger liquid cannot sustain the level of zero-point motion stronger than that corresponding to $\lambda_{0 s} \simeq 0.3896$ which is smaller than the dimer dissociation threshold $\lambda_{02}=\sqrt{2} / \pi \simeq 0.4502$.

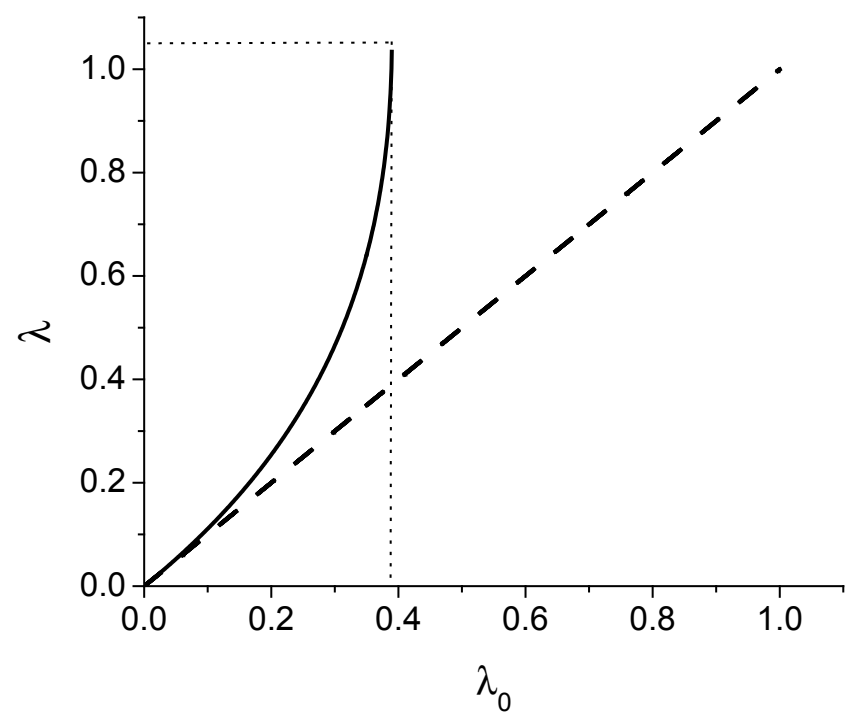

FIG. 3. The dependence of the Luttinger liquid quantum parameter $\lambda$, Eq.(17) on De Boer's number $\lambda_{0}$, Eq.(4). The dashed line is $\lambda=\lambda_{0}$.

The $\lambda\left(\lambda_{0}\right)$ dependence found by inverting Eq.(38) is shown in Fig.3 by a solid line. In the classical limit, $\lambda_{0} \rightarrow 0$, we have $\lambda \rightarrow \lambda_{0}$ as expected; then the effective pair potential (34) reduces to its bare Morse form. As $\lambda_{0}$ grows, the difference between $\lambda$ and $\lambda_{0}$ increases. We note that De Boer's number $\lambda_{0}(4)$ is always smaller than its Luttinger liquid counterpart $\lambda(17)$ which reflects softening of the Luttinger liquid by zero-point motion. The end point of the $\lambda\left(\lambda_{0}\right)$ dependence which is the limit of stability of the liquid phase is a critical phenomenon there the $\lambda\left(\lambda_{0}\right)$ dependence has an infinite slope. Upon approaching $\lambda_{0 s}$ from below we find that $\lambda$ is given by

$$
\lambda=\lambda_{s}-\left[\frac{2 e}{\ln (1+\pi / 2)}\left(\lambda_{0 s}-\lambda_{0}\right)\right]^{1 / 2}
$$

Although both the reduced rms fluctuation $f^{*}(35)$ and bond length $Q$ (36) are increasing functions of the Luttinger liquid parameter $\lambda(17)$, the relative fluctuation $f^{*} / Q$ has a maximum at $\lambda \ln (1+\pi / 2)=Q_{0} / 3$. While the discussion of specific molecular substances will be postponed until Section VI, here we note that in our attempts to fit real two-body potentials into the Morse form the reduced classical bond length was always found to satisfy $Q_{0} \gtrsim 5$. The relatively large value of $Q_{0}$ is a reflection of the strength of the short-distance overlap repulsion and weakness of the large-distance attraction in the pair interaction potential. With $Q_{0}=5$ (used hereafter for estimates) we find that $\lambda=(5 / 3) \ln ^{-1}(1+\pi / 2) \simeq 1.7651$ at the maximum of the relative fluctuation. But the Luttinger liquid cannot exist for $\lambda>\lambda_{s}=\ln ^{-1}(1+\pi / 2) \simeq$ 1.0591 . 
Therefore the relative fluctuation, $f^{*} / Q$, reaches its maximal value at the border of existence of the Luttiner liquid, $\lambda_{s} \simeq 1.0591$, with the magnitude not exceeding the level of about 0.18 . This fact resembling Lindemann's empirical criterion of melting [34] verifies that our description of the bond dynamics as due to coupling to a bath of harmonic oscillators is quantitatively correct. The inequality $f^{*} / Q \ll 1$ implies that underlying particles never come into close contact with each other - the effect of particle statistics is negligible. In addition, having $Q_{0}$ substantially larger than unity justifies the nearest-neighbor interaction approximation in our treatment of the liquid.

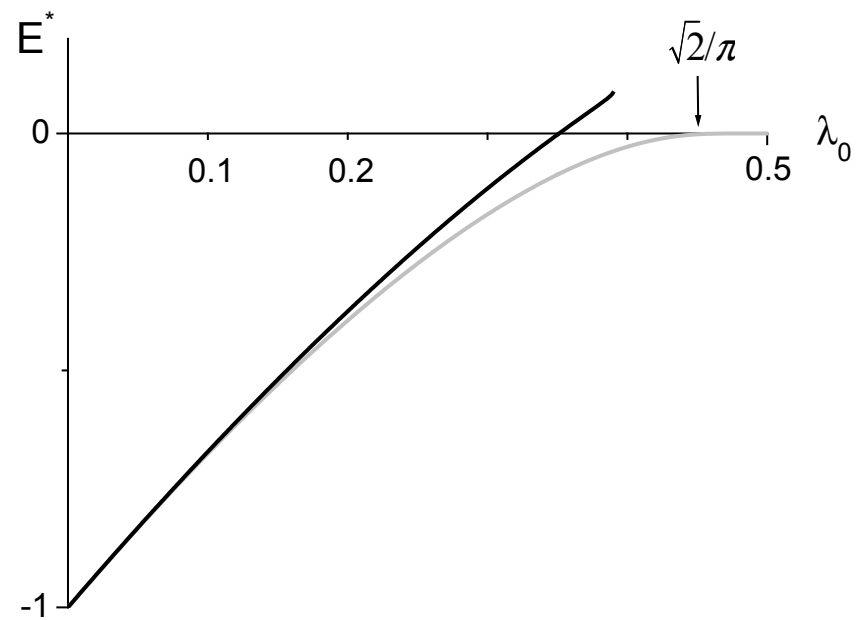

FIG. 4. The reduced energy per particle of the Luttinger liquid phase as a function of De Boer's number $\lambda_{0}$ (4). The lower grey scale curve corresponds to approximate solution described by Eqs.(26) and (27) while the upper curve is an accurate solution given by Eqs.(37) and (38). The arrow shows the location of the dimer dissociation threshold $\lambda_{02}=\sqrt{2} / \pi$.

The only remaining approximation which needs to be addressed is our replacement of the vibrational spectrum of the system, $\omega(k)=(2 c / H)|\sin (k H / 2)|$, by the Debye model, $\omega(k)=c|k|$, valid for $\omega \leq \omega_{D}=\pi c / H$. Both spectra are fairly close to each other: they coincide in the long-wavelength limit, $k H \ll 1$, and end at the edges of the first Brillouin zone, $k= \pm \pi / H$. Physically the chain with the Debye spectum is less susceptible to short-wavelength fluctuations than the original system - the Debye frequency $\omega_{D}$ is $\pi / 2$ times larger than the maximal allowed frequency. This difference necessary to have the correct number of degrees of freedom will only have a marginally small effect on final results because of the dominant role played in one dimension by low-energy long-wavelength fluctuations where the Debye approximation becomes exact. (a)

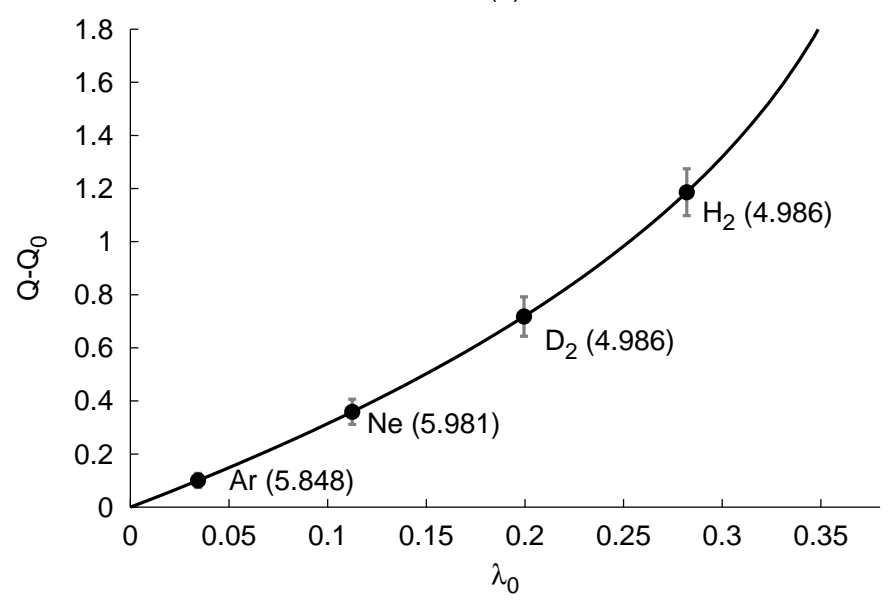

(b)

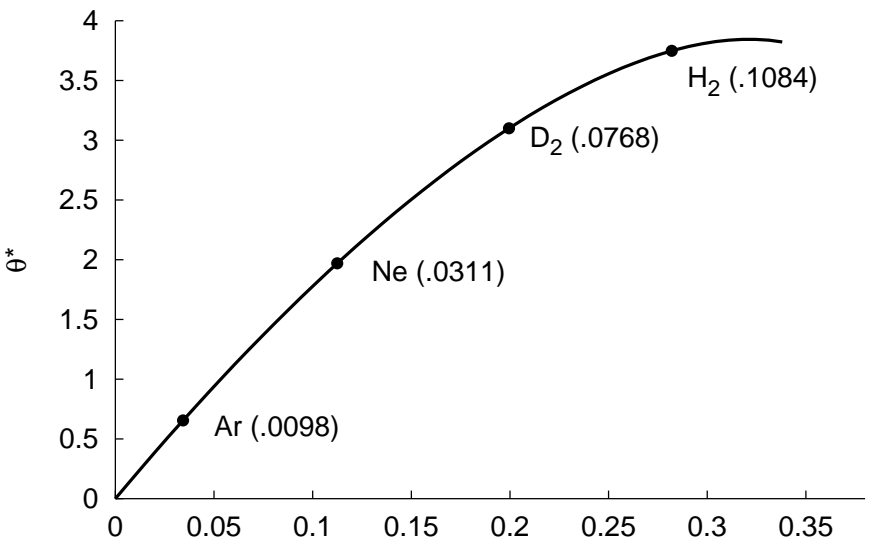

FIG. 5. The reduced quantuni ${ }^{\lambda}$ expansion $Q-Q_{0}$ (a), and the Debye temperature $\theta^{*}(\mathrm{~b})$ of the Luttinger liquid as functions of De Boer's number $\lambda_{0}(4)$. Various molecular substances are shown as solid dots on the curves. The numbers in the brackets on the quantum expansion graph are classical reduced bond lengths $Q_{0}$ while the size of vertical bars equals the relative bond fluctuation. The numbers in the brackets on the Debye temperature graph are Luttinger liquid exponents $g(11)$.

The upper curve of Fig. 4 shows the reduced groundstate energy per particle of the Luttinger liquid as a function of De Boer's number $\lambda_{0}$ (4) found by combining Eqs.(37) and (38). Qualitatively similar dependence was found in Ref. [19]; our energy is about 10 percent lower. For the purpose of comparison the lower grey scale curve of Fig.4 shows the approximate reduced energy per particle, Eqs.(26) and (27), which was argued to constrain the $E_{L L}^{*}\left(\lambda_{0}\right)$ dependence from below. The quantitative difference between the curves is not very large and becomes noticeable only for $\lambda_{0}>0.15$. Since variational analysis always constrains the ground-state energy from above, the true $E_{L L}^{*}\left(\lambda_{0}\right)$ dependence must be sandwiched 
between the curves of Fig.4. We expect however that $E_{L L}^{*}\left(\lambda_{0}\right)$ is well-approximated by Eqs.(37) and (38) (the upper curve of Fig.4).

Variation of other properties of the Luttinger liquid with De Boer's number $\lambda_{0}(4)$ can be readily calculated. The expression for the reduced sound velocity $c^{*}=c / c_{0}$ can be found to be

$$
\begin{aligned}
c^{*} & =\lambda_{0} Q / \lambda Q_{0} \\
& =e^{-\lambda \ln (1+\pi / 2)}\left[1+3\left(\lambda / Q_{0}\right) \ln (1+\pi / 2)\right],
\end{aligned}
$$

which together with (38) parametrically determine $c^{*}\left(\lambda_{0}\right)$. In the range of interest $Q_{0} \gtrsim 5$ the reduced sound velocity is a monotonically decreasing function of $\lambda_{0}$. At the boundary of existence of the Luttinger liquid the reduced sound velocity reaches its minimal (and finite) value and has a square-root singularity implied by Eq.(39).

Similarly the dependence of the Luttinger liquid exponent $g(11)$ on $\lambda_{0}$ (4) can be found by combining Eqs.(19), (36) and (38). Again for $Q_{0} \gtrsim 5$ the correlation exponent $g$ is a monotonically increasing function of $\lambda_{0}$ reaching its maximal value at the boundary of existence of the liquid phase.

The $\lambda_{0}$ dependence of the reduced Debye temperature $\theta^{*}$ is determined by

$$
\theta^{*}=2 \pi / v=2 \pi^{2} \lambda e^{-2 \lambda \ln (1+\pi / 2)},
$$

combined with Eq.(38). The right-hand side of (41) has a maximum at $\lambda=\lambda_{s} / 2=0.5 \ln ^{-1}(1+\pi / 2) \simeq 0.5295$ which is inside the range of existence of the liquid phase. In view of (38) this corresponds to De Boer's number $\lambda_{0}=1 /\left[2 e^{1 / 2} \ln (1+\pi / 2)\right] \simeq 0.3212$ which is the location of the maximum of the $\theta^{*}\left(\lambda_{0}\right)$ dependence.

Fig. 5 shows the dependence of the reduced quantum expansion $Q-Q_{0}$ and Debye temperature $\theta^{*}$ on De Boer's number $\lambda_{0}$. The quantum expansion is found by combination of Eqs.(36) and (38); it is merely a magnified $\lambda\left(\lambda_{0}\right)$ curve of Fig.3. The $\theta^{*}\left(\lambda_{0}\right)$ dependence (Fig.5b) is constructed by combining Eqs.(41) and (38). Both properties are linear functions of De Boer's number in the $\lambda_{0} \rightarrow 0$ limit.

\section{Zero-pressure phase diagram}

In order to construct the phase diagram of the system we need to compare all the branches of the reduced energy function (5); for given De Boer's number $\lambda_{0}$ the branch with lowest $E^{*}\left(\lambda_{0}\right)$ singles out the ground-state. The outcome is shown in Fig.6 where the reduced energy per particle of the Luttinger liquid, Eqs.(37) and (38), is drawn together with those for diatomic, Eq.(7), and monoatomic, $E_{\text {mono }}^{*}\left(\lambda_{0}\right)=0$, gases.
We see that as $\lambda_{0}$ increases away from the classical limit, $\lambda_{0}=0$, the reduced energy per particle of the Luttinger liquid increases, and at $\lambda_{0 e v} \simeq 0.3365$ a crossing with the dimer gas energy curve, Eq.(7), takes place liquid phase evaporates into diatomic gas via discontinuous transition. We note that the Luttinger liquid can still coexist (as a metastable state) with gas phases in the narrow range $0.3365 \lesssim \lambda_{0} \lesssim 0.3896$. This is shown by the grey scale part of the energy curve.

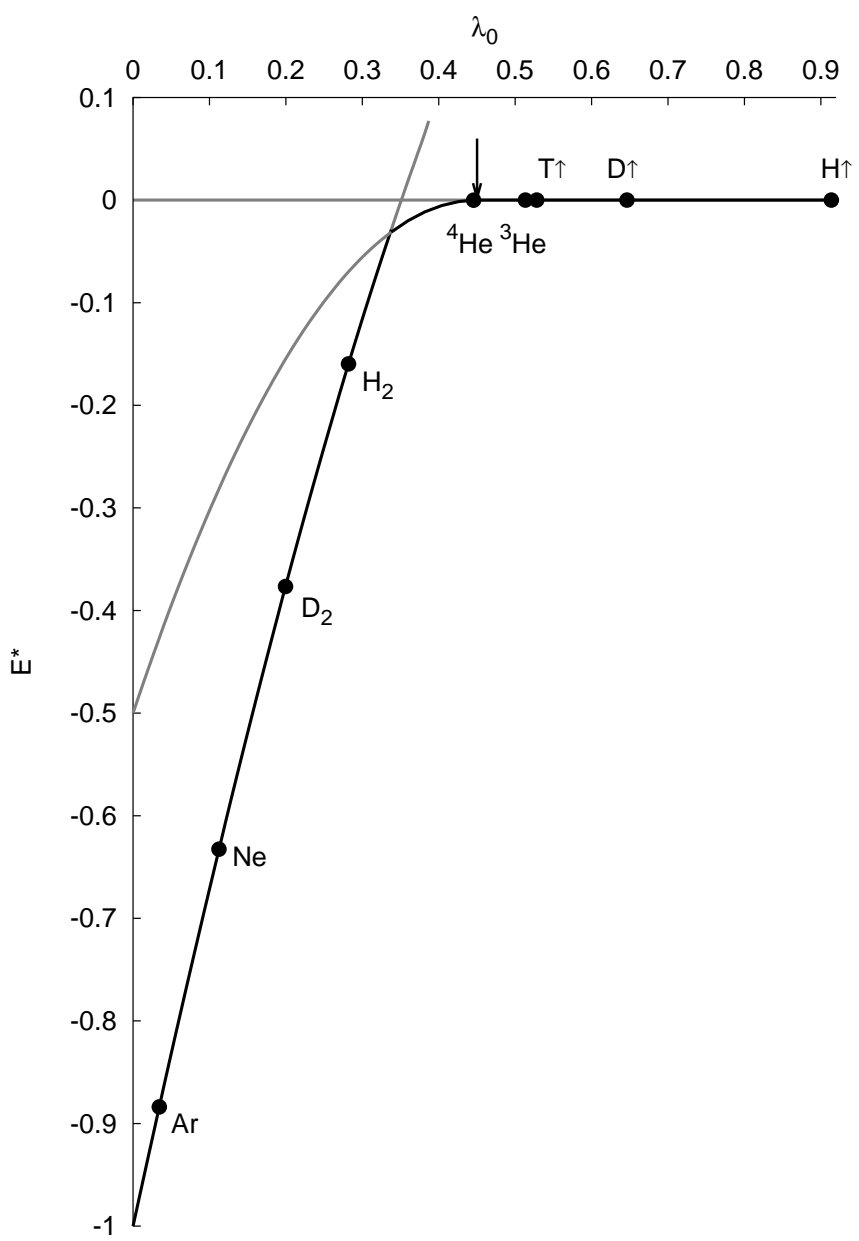

FIG. 6. The dependencies of the reduced energy per particle on De Boer's number $\lambda_{0}$ (4) for various phases. The bold parts of the curves correspond to ground states of the system while the grey scale segments indicate metastable states. The loci of a series of substances are shown by solid dots, and the arrow pointing down is the dimer dissociation threshold.

The diatomic gas is the ground-state of the system in the range $0.3365 \lesssim \lambda_{0}<\sqrt{2} / \pi \simeq 0.4502$ : at $\lambda_{02}=\sqrt{2} / \pi$ it undergoes a continuous dissociation transition into a monoatomic gas. For $\lambda_{0}>\sqrt{2} / \pi$ quantum fluctuations are too strong and no bound state can exist: a monoatomic gas is the only possible state of the system.

In determining the ground-state one needs to take into account all possible competing phases of the system 
in question. We however neglected the possibility that trimers (and generally $N$-atomic molecules) may come into play. This is because we only know how to treat the $N=1,2, \infty$ cases in a controlled fashion. Although it appears unlikely, we cannot rule out that for sufficiently large $\lambda_{0}, N>2$-atomic gases might become relevant; resolving this issue is left for future study.

A critical reader still may argue that our prediction of the diatomic gas phase is an artifact of the variational treatment - the exact energy curve corresponding to the Luttinger liquid may go lower then what Fig.6 shows. If this is the case, then the dimer gas ground state may disappear altogether.

A finite-pressure treatment described next provides additional evidence that physics is incomplete with only liquid and monoatomic gas present.

\section{FINITE PRESSURE}

At zero pressure there is a qualitative difference between a liquid which is a bound many-body state and a gas which is a collection of infinitely far separated particles. Arbitrarily small confining pressure necessarily brings a gas to a finite density. As a result the two gas phases previously discussed turn into Luttinger liquids. The difference between the "parent" Luttinger liquid and what used to be a monoatomic gas becomes merely quantitative - they will have differing densities, sound velocities, correlation exponents and other properties. The liquid of dimers is more complicated as in addition its oscillation spectrum will have an extra optical branch.

To avoid confusion we will keep referring to these pressure-induced Luttinger liquids as gases. As the pressure and De Boer's number $\lambda_{0}$ change, they may undergo gas-gas and gas-liquid transitions. At sufficiently large pressure and $\lambda_{0}$ the quantitative difference between the gases and the liquid must disappear.

In what follows we will not be able to discuss these transitions as at the moment it is unclear how to describe the effect of pressure on the diatomic gas phase in a controlled fashion. On the other hand, generalization of our formalism to monoatomic gas and Luttinger liquid is straightforward. Thus we will restrict ourselves to finding ranges of existence of these phases.

The difference between the liquid and gas becomes most extreme at negative pressure. Here the liquid may still exist as a metastable state while the gas phase is impossible.

In one dimension the pressure has dimensionality of a force. Assume our system is compressed by a constant force $p$ applied to its ends. The system responds by exerting an outward force of magnitude $p$ on the compressing agent which corresponds to the definition of positive pressure. Similarly, if the system is stretched by an external force, it responds by exerting an inward force on the stretching agent which corresponds to the definition of negative pressure. These two cases will be distinguished by the sign of $p$.

Since every particle of the system is in mechanical equilibrium, the whole effect of pressure translates into replacing the bond potential $V(h)$ by $V(h)+p h$, the total potential energy in the external field [23]. Then the finite-pressure analog of Eq.(20) becomes

$$
\begin{aligned}
E^{*}(\gamma, Q) & =v^{-1} \ln (1+\gamma)-2 e^{Q_{0}-Q}\left(1+\gamma^{-1}\right)^{\lambda} \\
& +e^{2\left(Q_{0}-Q\right)}\left(1+\gamma^{-1}\right)^{4 \lambda}+p^{*} Q,
\end{aligned}
$$

where $p^{*}=p l / \epsilon$ is the reduced pressure. Eq.(42) should be minimized with respect to $\gamma$ and $Q$, and then $v=$ $\lambda / \pi \lambda_{0}^{2}$ and $\gamma=2 / \pi$ substituted in the outcome will guarantee translational invariance. The results are finite pressure analogs of Eqs.(34), (36), and (38):

$$
\begin{aligned}
E^{*}(Q) & =\left(\pi \lambda_{0}^{2} / \lambda\right) \ln (1+2 / \pi)-2 e^{Q_{0}-Q+\lambda \ln (1+\pi / 2)} \\
& +e^{2\left(Q_{0}-Q\right)+4 \lambda \ln (1+\pi / 2)}+p^{*} Q \\
Q= & Q_{0}+3 \lambda \ln (1+\pi / 2) \\
- & \ln \left[\left(1+\sqrt{1+2 p^{*} e^{2 \lambda \ln (1+\pi / 2)}}\right) / 2\right] \\
\lambda_{0} & =\lambda e^{-\lambda \ln (1+\pi / 2)}\left[\left(1+2 p^{*} e^{2 \lambda \ln (1+\pi / 2)}\right.\right. \\
& +\sqrt{\left.\left.1+2 p^{*} e^{2 \lambda \ln (1+\pi / 2)}\right) / 2\right]^{1 / 2}}
\end{aligned}
$$

The expressions for the reduced rms fluctuation (35) and Debye temperature (41) as functions of $\lambda$ remain the same while the $\lambda\left(\lambda_{0}\right)$ dependence is determined by (45). Similarly the reduced sound velocity is given by the first representation of Eq.(40) with $Q$ and $\lambda$ determined by Eqs.(44) and (45), respectively.

We note that the parameter $\lambda(17)$ now accounts for both the effects of pressure and zero-point motion.

\section{A. Classical limit}

In the classical limit $\lambda_{0}, \lambda \rightarrow 0$ and $p^{*}>0$ the position of the minimum of (43) given by (44) naturally shifts to values smaller than $Q_{0}$. In addition Eq.(44) predicts that at a very large pressure $p_{0}^{*} \simeq 2 e^{2 Q_{0}}=2 e^{10} \simeq 44000$ the bond length vanishes. This conclusion is an artifact because for small interparticle separation the Morse potential underestimates the true strength of overlap repulsion - the bond length can only go to zero in the limit of infinite pressure. This flaw implies that only $p^{*} \ll p_{0}^{*}$ results are credible which is not really restrictive as $p_{0}^{*}$ is unrealistically large.

The pressure dependence of the reduced sound velocity follows from the first representation of (40), and Eqs.(44) and (45).

For not very large negative pressure the pair potential (43) has a minimum given by (44) and a maximum - the 
"broken" ground state of the chain is separated from the metastable stretched crystal by a potential barrier. As the magnitude of the pressure increases, the amplitude of the barrier decreases, and at the classical limit of mechanical stability, $p_{m s}^{*}=-1 / 2$, the barrier disappears altogether - no bound state can exist for $p^{*}<-1 / 2$. As the pressure approaches the limit of mechanical stability from above, the reduced bond length tends to $Q=Q_{0}+\ln 2$ while the reduced sound velocity vanishes as $\left(1+2 p^{*}\right)^{1 / 4}$ in agreement with general arguments of Ref. [23].

\section{B. Quantum case}

With quantum effects included, the pressure at which the bond length vanishes (and the Morse potential approximation fails) will be even larger than its classical counterpart as zero-point motion counteracts the compression. Therefore the restriction $p^{*} \ll p_{0}^{*}$ remains unchanged in the quantum case.

Since quantum fluctuations lead to the expansion and softening of the liquid phase, a smaller in magnitude negative pressure will suffice to destabilize the liquid - the dependence of the limit of mechanical stability on De Boer's number (4), $p_{m s}^{*}\left(\lambda_{0}\right)$, should be a monotonically increasing function of $\lambda_{0}$ satisfying $p_{m s}^{*}(0)=-1 / 2$ (classical limit) and $p_{m s}^{*}\left(\lambda_{0 s}\right)=0$ (zero-pressure limit of stability of the Luttinger liquid).

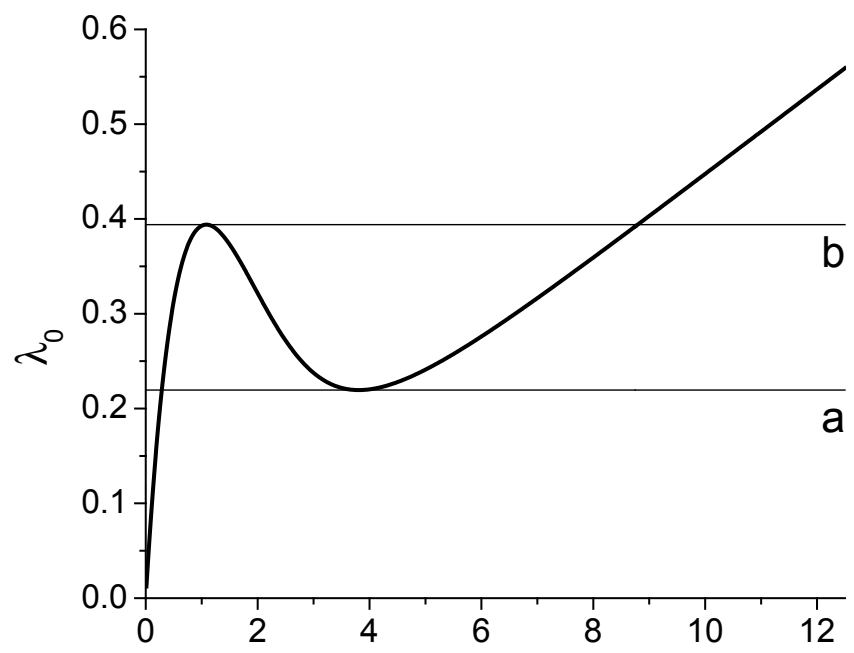

FIG. 7. The $\lambda_{0}(\lambda)$ dependencA, Eq.(45), for suffifciently small positive pressure $\left(p^{*}=0.002\right.$ is shown). The lines of fixed $\lambda_{0}=\lambda_{0 a, b}$ are the limits of stability of gas and liquid, respectively.

For $p^{*}=0$ the right-hand-side of (45) vanishes both at $\lambda=0$ and $\lambda=\infty$ reaching a maximum at $\lambda=\lambda_{s}$ which determined the limit of stability of the liquid in the zero-pressure case (see Section IVC). For finite positive pressure and $\lambda \rightarrow \infty$ the right-hand-side of (45) behaves as $p^{* 1 / 2} \lambda$ which implies that for not very large $p^{*}$ the $\lambda_{0}(\lambda)$ dependence is a nonmonotonic function which has both a maximum and a minimum. The position of the minimum shifts to infinity as $p^{*} \rightarrow 0$. An example of the $\lambda_{0}(\lambda)$ dependence, Eq.(45), for sufficiently small positive pressure is displayed in Fig. 7 where we also show two lines of constant $\lambda_{0}$ to help identify possible phases of the system.

For fixed small positive pressure and $\lambda_{0}<\lambda_{0 a}$ Eq.(45) has a unique solution for $\lambda$ describing the liquid. For $\lambda_{0 a}<\lambda_{0}<\lambda_{0 b}$ Eq.(45) has three solutions. Out of them only the smallest (corresponding to liquid) and the largest (corresponding to gas) are physical. For $\lambda_{0}>\lambda_{0 b}$ there is only one solution for $\lambda$ describing a gas phase. The liquid and gas phases can coexist in the range of $\lambda_{0}$ between the limit of existence of the gas phase, $\lambda_{0 a}$, and that of liquid, $\lambda_{0 b}$.

If the condition

$$
p^{*} \exp \left[\left(2 \lambda_{0} / p^{* 1 / 2}\right) \ln (1+\pi / 2)\right] \gg 1
$$

holds, then the explicit $\lambda_{0}$-dependence of the properties of the gas phase can be deduced from Eqs.(44) and (45)

$$
\begin{gathered}
\lambda=\lambda_{0} / p^{* 1 / 2} \\
Q-Q_{0}=-(1 / 2) \ln \left(p^{*} / 2\right) \\
+\frac{1}{\sqrt{p^{*}}}\left[2 \lambda_{0} \ln \left(1+\frac{\pi}{2}\right)-\frac{1}{\sqrt{2}} e^{-\left(\lambda_{0} / p^{* 1 / 2}\right) \ln \left(1+\frac{\pi}{2}\right)}\right]
\end{gathered}
$$

We note the range of applicability of these results is rather wide - small pressure and nonzero $\lambda_{0}$, large pressure and arbitrary $\lambda_{0}$, and arbitrary pressure and large $\lambda_{0}$. The pressure dependence of the reduced length per particle $Q$ (48) is the equation of state of the Morse gas.

As the pressure increases, the distance between the minimum and maximum of the right-hand-side of (45) decreases, and at the critical pressure $p^{*}=p_{c}^{*} \simeq 0.0185$ the difference between the properties of liquid and gas disappears for the first time. At this pressure and $\lambda_{0 c} \simeq$ 0.4387 the size of the liquid-gas coexistence region shrinks to a point.

The negative pressure analysis is similar to what we did for $p^{*}=0$ in Section IVC. For negative pressure of sufficiently small magnitude the right-hand-side of Eq.(45) vanishes both at $\lambda=0$ and $1+2 p^{*} e^{2 \lambda \ln (1+\pi / 2)}=0$ reaching a maximum in between. If De Boer's parameter $\lambda_{0}$ is below this maximum, then Eq.(45) has two solutions for $\lambda$. The smaller (physical) solution describes a metastable Luttinger liquid. As $\lambda_{0}$ increases, the two solutions approach each other. When $\lambda_{0}$ reaches the maximum of the right-hand-side of (45), we are at the limit of mechanical stability of the system - no liquid can exist for larger $\lambda_{0}$.

Alternatively, for sufficiently small fixed $\lambda_{0}$ the height of the maximum of (45) decreases upon increase of the magnitude of pressure, and at some $p_{m s}^{*}\left(\lambda_{0}\right)$ the maximum of (45) reaches the level of $\lambda_{0}$ thus bringing the 
system to the limit of mechanical stability. It is curious that in the quantum case the "liquid" solution disappears before the condition $1+2 p^{*} e^{2 \lambda \ln (1+\pi / 2)}=0$ is reached. Therefore at the stability threshold both the energy barrier (between "broken" ground-state and stretched metastable liquid) and sound velocity remain finite. Only in the classical limit $\lambda_{0} \rightarrow 0$ do these quantities vanish.

The pressure-De Boer's parameter diagram showing ranges of existence of liquid and gas is displayed in Fig.8.

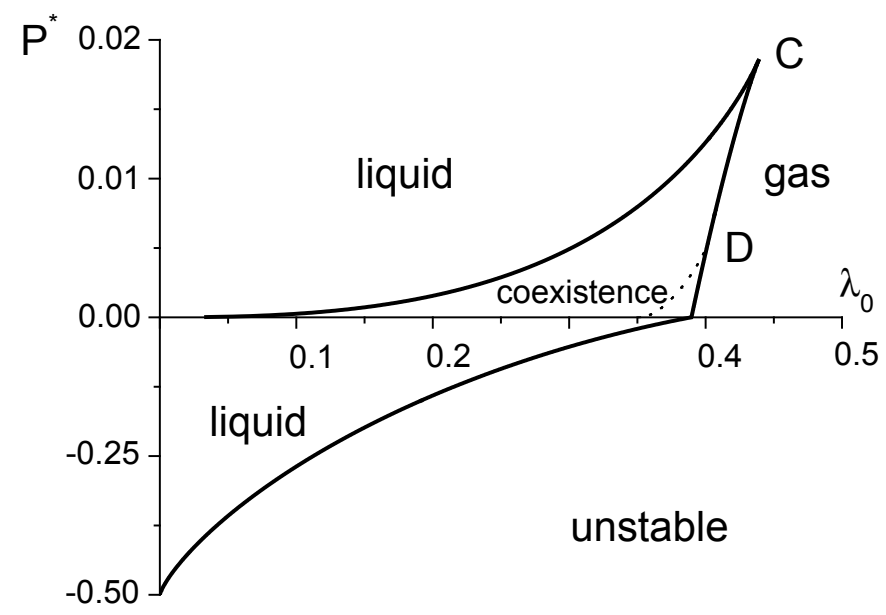

FIG. 8. The $p^{*}\left(\lambda_{0}\right)$ diagram showing ranges of existence of liquid and monoatomic gas phases. Different scales are selected on the positive and negative parts of the pressure axis. The liquid and gas can be in equilibrium along the dotted line.

We deliberately selected different scales on the positive and negative parts of the pressure axis in order to be able to show the complete picture. As a result of this choice there is an illusory change of slope of the line of mechanical stability of liquid at zero pressure - in reality the $p_{m s}^{*}\left(\lambda_{0}\right)$ dependence is smooth.

The point $\mathrm{C}$ having coordinates $\lambda_{0 c} \simeq 0.4387, p_{c}^{*} \simeq$ 0.0185 where the limits of existence of liquid and gas meet is a candidate for the liquid-gas critical point. Then the line of a liquid-gas evaporation transition should also pass through C. This curve, found by equating the groundstate energy (43) for both phases is shown in Fig.8 by a dotted line. It does not end at $\mathrm{C}$, and everywhere within the DC segment of the metastability line the Luttinger liquid has lower energy than the gas. These results imply that a direct liquid-monoatomic gas transition is impossible. Another gas phase, diatomic, must intervene. Although the existence of this phase will set phase boundaries at finite pressure, it will not affect the ranges of existence of the Luttinger liquid and monoatomic gas.
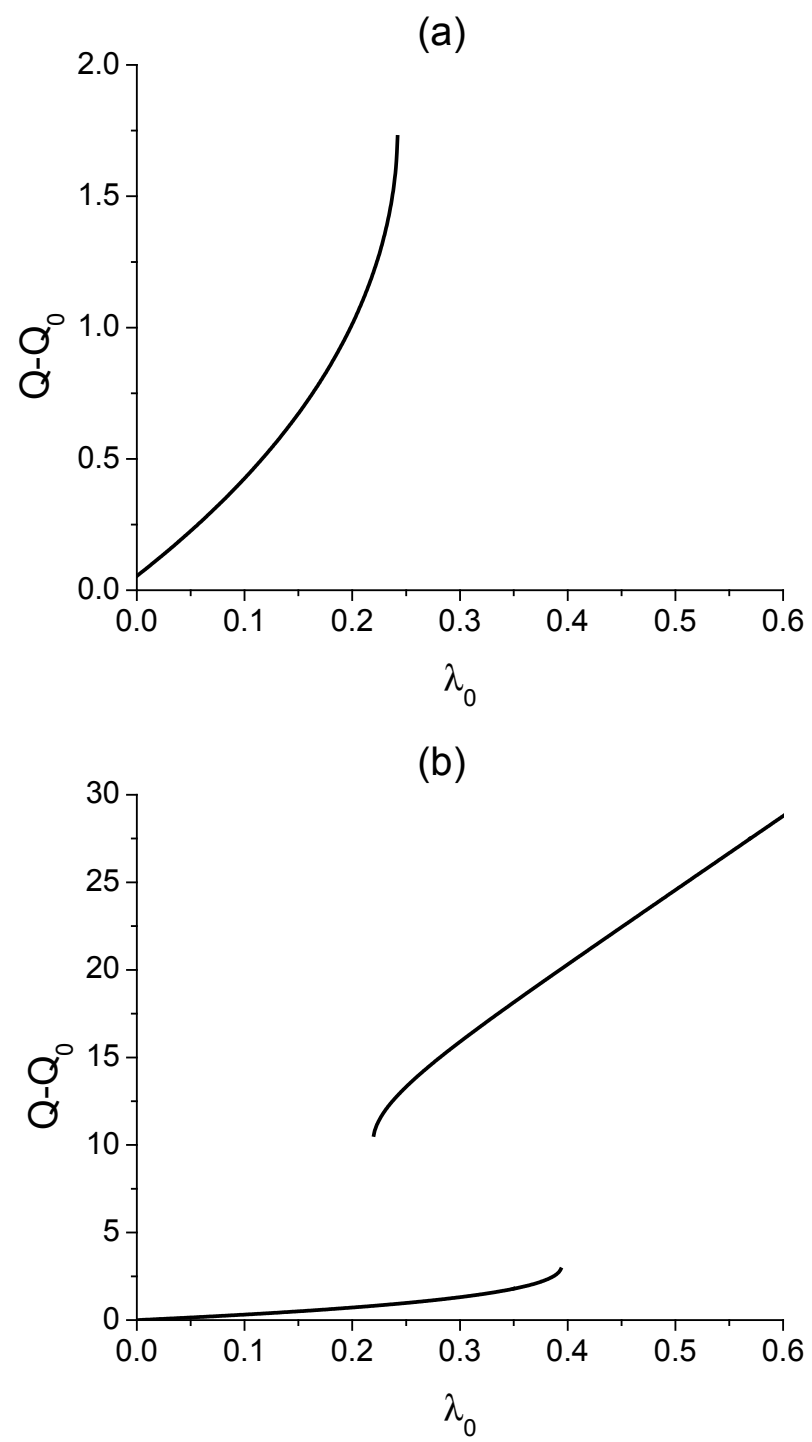

(c)

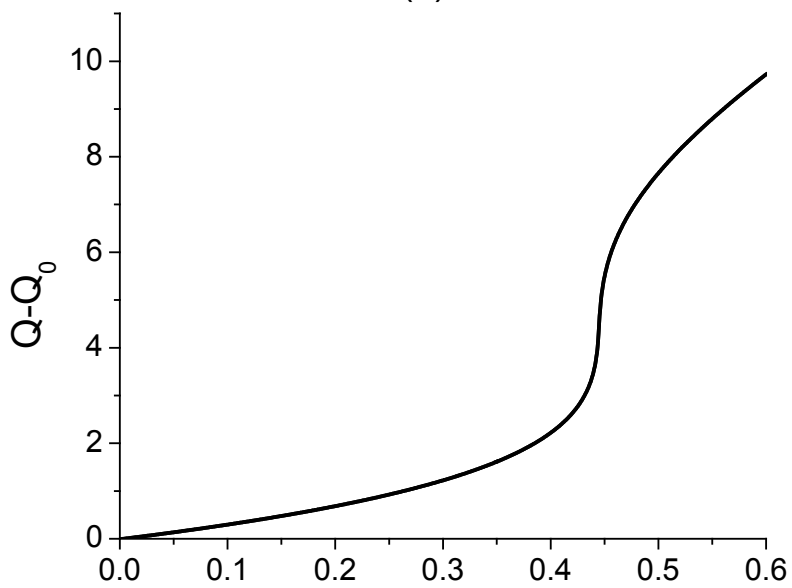

FIG. 9. The dependence of thareduced quantum expansion $Q-Q_{0}$ on De Boer's parameter $\lambda_{0}$ at a negative pressure of large magnitude (a), within the liquid-gas coexistence region (b), and past the point C of Fig.8 (c). 
Other finite pressure properties of the system can be readily found. As an example, Fig.9 illustrates the dependence of the reduced quantum expansion $Q-Q_{0}$ on De Boer's number $\lambda_{0}$ for three different pressures. The curve of Fig.9a shows the expansion of the Luttinger liquid due to the combined effect of zero-point motion and negative pressure of sufficiently large magnitude $\left(p^{*}=-0.1\right.$ was used). The end point of the dependence is the limit of mechanical stability of the liquid. In Fig.9b the pressure is selected to be within the region of the liquid-gas coexistence $\left(p^{*}=0.002\right.$ was used). As a result the quantum expansion dependence is not unique: the lower (larger density) curve corresponds to the liquid while the upper (lower density) curve curve is for the monoatomic gas. The end of the "liquid" curve and the beginning of the "gas" curve are limits of existence of these phases. In Fig.9c the pressure is selected to satisfy the condition $p^{*}>p_{c}^{*}$ i.e. past the point $\mathrm{C}$ of Fig.8. Now there is no quantitative difference between liquid and gas. The presence of a relatively steep part around $\lambda_{0} \simeq 0.44$ is the effect of the proximity of $p^{*}=0.02$ to $p_{c}^{*} \simeq 0.0185$.

To test the limits of applicability of our theory we also investigated the reduced sound velocity and relative bond fluctuation at various pressures. We found that at fixed $\lambda_{0}$ the reduced sound velocity initially increases with pressure but then at pressures exceeding the level of about 6000 or larger, it begins to decrease vanishing at the point where the bond length $Q$ vanishes. The fall and vanishing of the sound velocity do not correspond to physical reality and have their origin in inadequacy of the Morse potential approximation at small interparticle distances. Similarly, the relative fluctuation diverges upon approaching the nonphysical $Q=0$ point. These artifacts do not pose practical limitations to our theory because they occur at unrealistically large pressures. We verified that if we limit ourselves to pressures not exceeding 5000, then the relative fluctuation is smaller than 0.5, and the behavior of other properties of the system is in agreement with physical expectations.

TABLE I. Morse parameters for a series of molecular substances and some of their computed properties at zero pressure. Substances are arranged in the order of decrease of their De Boer's number $\lambda_{0}$. Blank entries correspond to gas ground states when interparticle separation is infinite.

\begin{tabular}{ccccccccc}
\hline \hline & $m$ & $\epsilon$ & $\ell$ & $H_{0}$ & $\lambda_{0}$ & $Q-Q_{0}$ & $Q$ & $-E *$ \\
& a.u. & $\mathrm{K}$ & $\AA$ & $\AA$ & & & & $10^{-3}$ \\
\hline$H \uparrow$ & 1.008 & 6.19 & .6869 & 4.153 & .9136 & & & 0 \\
$D \uparrow$ & 2.014 & 6.19 & .6869 & 4.153 & .6463 & & & 0 \\
$T \uparrow$ & 3.016 & 6.19 & .6869 & 4.153 & .5281 & & & 0 \\
${ }^{3} \mathrm{He}$ & 3.016 & 10.8 & .5350 & 2.980 & .5134 & & & 0 \\
${ }^{4} \mathrm{He}$ & 4.003 & 10.8 & .5350 & 2.980 & .4456 & & & .0509 \\
$\mathrm{H}_{2}$ & 2.016 & 32.2 & .6900 & 3.440 & .2819 & 1.1860 & 6.172 & 159.6 \\
$\mathrm{D}_{2}$ & 4.028 & 32.2 & .6900 & 3.440 & .1994 & .7178 & 5.703 & 376.6 \\
$\mathrm{Ne}$ & 20.18 & 35.6 & .5200 & 3.110 & .1124 & .3591 & 6.340 & 632.6 \\
$\mathrm{Ar}$ & 39.95 & 120 & .6600 & 3.860 & .0343 & .1005 & 5.949 & 883.9 \\
\hline \hline
\end{tabular}

\section{APPLICATIONS AND DISCUSSION}

Fitting two-body potentials of molecular substances into the Morse form can provide us with the depth of the potential well $\epsilon$, the interaction range $l$, and the position of the potential minimum $H_{0}$. Supplemented by the masses of the underlying particles, this information is an input of our theory which then allows to determine De Boer's quantum parameter $\lambda_{0}$ (4), the ground state and virtually any property.

The fitting procedure can introduce uncertainties because real two-body interactions do not have the Morse form. We already know that the Morse potential underestimates the strength of the overlap repulsion at short distances but as long as the reduced pressure does not exceed 5000, this flaw is practically irrelevant.

The Morse potential also underestimates the magnitude of Van der Waals attraction at large distances. We found that in the condensed state this shortcoming can be kept under the control by carrying out a Morse potential fit in a range of interparticle distances followed by a consistency check verifying that the segment of most probable particle location (formed by computed equilibrium bond length plus/minus its rms fluctuation) is well inside the fitting range.

The first four columns of Table I represent Morse parameters of various molecular substances which are used to compute De Boer's number $\lambda_{0}$ shown in the fifth column. The remaining three columns are reduced quantum expansion $Q-Q_{0}$, bond length $Q$, and the energy per particle $E^{*}$ (all at zero pressure) calculated using the theory developed in this paper. The loci of all these substances are also indicated on the reduced energy curve $E^{*}\left(\lambda_{0}\right)$ of Fig.6. For the substances whose ground state is the Luttinger liquid, Fig.5a also shows the magnitudes of the reduced classical bond length $Q_{0}$ and its relative fluctuation $f^{*} / Q$, while Fig.5b gives the values of the Luttinger liquid exponent $g$.

In computing these properties we also assumed that the three-dimensional form of the interaction does not change upon one-dimensional confinement of the particles and that translational symmetry is preserved. Both these assumptions are approximations if the confinement is achieved in carbon nanotube bundles because interparticle interaction is mediated by the carbon environment [35] while the axial motion takes place in a periodic potential [15]. The former effect generally weakens interparticle attraction at large distances thus making the system more quantum. On the other hand the external periodic potential due to the carbon environment has an opposite effect leading to upward renormalization of the mass. Therefore the properties of strictly onedimensional matter may differ qualitatively from those of the matter inside nanotube bundles. The effect of an axial periodic potential may be even more dramatic, and 
the effective mass approximation insufficient if the corrugation is strong enough to introduce a commensurateincommensurate phase transition [36]. This potentially important effect is beyond the scope of our theory and cannot be discussed here.

Before considering individual substances it is useful to look at the properties of one-dimensional matter as a whole and compare them with those of laboratory substances. The corresponding states analysis of threedimensional molecular matter is based on the LennardJones pair potential and except for the vicinity of the classical limit $\lambda_{0}=0$, it is empirical [2-4].

The substances in Table I are arranged in the order of decrease of their De Boer's number $\lambda_{0}$ which is naturally the same as in three dimensions. The main qualitative difference from the ordinary substances occurs because of the dominant role played by zero-point motion which in one dimension forbids the crystal ground state. We find that spin-polarized isotopes of hydrogen (hydrogen $H \uparrow$, deuterium $D \uparrow$, and tritium $T \uparrow$ ) and ${ }^{3} \mathrm{He}$ are monoatomic gases, ${ }^{4} \mathrm{He}$ is diatomic gas, while molecular hydrogen and heavier substances are Luttinger liquids. If we view the Luttinger liquid as the counterpart of the crystal in three dimensions, then the bold part of the reduced energy curve, Fig.6, closely resembles its threedimensional counterpart [3]. There is a change of slope somewhere between $\mathrm{H}_{2}$ and ${ }^{4} \mathrm{He}$ which in our case is dissociation of the Luttinger liquid into a diatomic gas while in the three-dimensional world it is a melting transition.

Our dependence of the reduced Debye temperature $\theta^{*}$ on De Boer's number $\lambda_{0}$, Fig. 5b, also looks very similar to its three-dimensional counterpart [2], and even empirical values of the reduced Debye temperature are close to their computed one-dimensional analogs. We also find that $\theta^{*}\left(\lambda_{0}\right)$ dependence has a maximum somewhere past molecular hydrogen; from empirical data it seems impossible to tell whether this effect is present or not in three dimensions.

Before comparing quantum expansion in one and three dimensions, we note that the quantum theorem of the corresponding states applied to the Lennard-Jones system predicts that the reduced volume per particle (length in one dimension) $Q$ is only determined by De Boer's quantum parameter $\lambda_{0}$. At the same time for the Morse system the analogous statement is valid for the reduced quantum expansion $Q-Q_{0}$. However the inspection of Table I (see also Fig. 5b) shows that the values of the reduced classical bond length $Q_{0}=H_{0} / l$ belong to the relatively narrow interval roughly between 5 and 6.3 . Therefore the variation of $Q_{0}$ from substance to substance can be ignored and within experimental error our results can be compared to their empirical counterparts $[2,3]$. Again we find that the $\lambda_{0}$ dependencies of the reduced quantum expansion in one and three dimensions are qualitatively similar.

In three dimensions all these properties can be com- puted perturbatively in the $\lambda_{0} \rightarrow 0$ limit $[2,3]$ with the conclusion that to leading order $E^{*}\left(\lambda_{0}\right)-E^{*}(0), Q-Q_{0}$, and $\theta^{*}$ all vanish linearly with $\lambda_{0}$. This behavior is identical to the $\lambda_{0} \rightarrow 0$ limit of our theory.

As a final comment, we note that from the viewpoint of their electron transport properties all the molecular substances are normally insulators as they have completely filled electronic shells. However at sufficiently large pressure when electron wave functions of neighboring molecules overlap considerably, any substance should turn into a metal [37]. At that point our "molecular" approximation describing many-body physics in terms of additive two-body interactions fails. Typically this happens at a very large pressure, and a different approach explicitly accounting for the dynamics of the electron degrees of freedom is necessary. This complex problem is beyond the scope of the present paper. For the case of molecular hydrogen, however, we will be able to estimate this critical pressure when the metal-insulator transition takes place without leaving the framework of our theory.

In subsequent discussion of individual substances we first present the results based on the pair interaction potential in free space. These conclusions are robust. On the other hand, our comments about the properties of matter inside nanotubes are speculative as they rely on the assumption that the effect of carbon environment can be accommodated within the framework of our theory by adjusting De Boer's parameter $\lambda_{0}$. It is also important to keep in mind that inside nanotube bundles there will be an additional interaction between different onedimensional channels filled with absorbed substances. This interaction is responsible for exotic crossover effects which can be viewed as an effective change of space dimensionality [38]. These effects are also beyond the scope of our one-dimensional theory.

\section{A. Spin-polarized hydrogen and its isotopes}

The pair interaction between two particles of the spinpolarized hydrogen family has been computed by Kolos and Wolniewicz [39]. Etters, Dugan and Palmer [40] have found a very good Morse fit to the Kolos-Wolniewicz potential; the Morse parameters shown in Table I are their values.

Compared to the other elements in Table I, these substances have the shallowest potential well which is only $6.19 K$ deep. Combined with its smallest mass, this makes $H \uparrow$ the "quantummost" element with $\lambda_{0}=0.9136$. Spin-polarized deuterium $D \uparrow$ is second in line with $\lambda_{0}=0.6463$ while spin-polarized tritium $T \uparrow$ takes the third place, $\lambda_{0}=0.5281$. All these elements are monoatomic gases at zero pressure as can be seen from Fig.6. On the other hand at zero pressure in three dimensions the heaviest of the family, $T \uparrow$, forms a liquid while $H \uparrow$ and $D \uparrow$ are gases [40]. 
External pressure confines these gases to a finite density but because De Boer's numbers are larger than $\lambda_{0 c} \simeq 0.4387$ corresponding to point $\mathrm{C}$ of Fig.8, applying pressure is not going to turn them into liquids.

\section{B. Helium}

The pair interaction between two helium atoms is accurately described by the semi-empirical Aziz potential [41] which is $10.8 \mathrm{~K}$ deep; this is the second entry in Table I. The authors of Ref. [19] proposed the Morse fit of the Aziz potential with the parameters $l=0.5828 \AA$ and $H_{0}=2.89 \AA$ claiming that "the integrated square of the deviation of the fit from the Aziz potential does not exceed $1 \%$ in the range of localization of a $\mathrm{He}$ atom". These parameters produce $\lambda_{0} \simeq 0.41$ for ${ }^{4} \mathrm{He}$ which according to our theory makes it a diatomic gas. It is indeed experimentally known [42] that ${ }^{4} \mathrm{He}$ can form very large dimers with the bond length of $52 \stackrel{\circ}{A}$. We verified however that at interparticle distances that large the Morse fit proposed in Ref. [19] is very poor.

Our own attempts to improve the fit increased the value of $\lambda_{0}$ bringing it into a narrow vicinity of the dimer dissociation threshold $\lambda_{02}=\sqrt{2} / \pi$, and without extra knowledge we could not make a decision whether $\lambda_{0}$ is larger or smaller than $\lambda_{02}$. We resolved this dilemma by invoking the experimental result [42] that the binding energy of the ${ }^{4} \mathrm{He}$ dimer is $-1.1 \mathrm{mK}$. Halving this value and dividing the outcome by $10.8 K$, the depth of the $H e-H e$ potential well, produces the last entry in Table I, the reduced energy per particle of the diatomic gas. This can be substituted into Eq.(7) to recover the fifth entry, $\lambda_{0}=0.4456$. As expected, this is only marginally smaller than the dimer dissociation threshold $\lambda_{02}$. Using the definition of De Boer's number, Eq.(4), we can now recover the interaction range (the third entry in Table I) to be $l=0.5350 \AA$. Finally the position of the minimum of the Morse potential $H_{0}=2.980 \AA$ was chosen to optimize the fit.

Upon application of pressure the gas of ${ }^{4} \mathrm{He}$ dimers will turn into a Luttinger liquid; calculation of the properties of this dimer liquid is beyond the scope of our method.

De Boer's quantum parameter for ${ }^{3} \mathrm{He}$ can be obtained from that for ${ }^{4} \mathrm{He}$ by invoking their mass ratio. This gives us the value quoted in the fifth column of the ${ }^{3} \mathrm{He}$ row in Table I. It is higher than the dimer dissociation threshold $\lambda_{02}$ thus ruling out earlier prediction [43] that ${ }^{3} \mathrm{He}$ can form a dimer in one dimension. A many-body system of ${ }^{3} \mathrm{He}$ particles in one dimension will form a monoatomic gas with properties close to those of spinpolarized tritium. Similar to $T \uparrow$, the ${ }^{3} \mathrm{He}$ gas will not condense under pressure.

Our result that ${ }^{4} \mathrm{He}$ forms a diatomic gas strictly in one dimension is in variance with earlier work $[15,16,18]$ which predicted a liquid ground state with the binding energy of order a few to tens $m K$. This is the same order of magnitude as the energy per particle in the diatomic gas. However Refs. [16,18] also predict a liquidsolid phase transition which is forbidden in one dimension.

In applying our results to nanotubes one has to bear in mind that ${ }^{4} \mathrm{He}$ atoms are strongly attracted to the interstitial channels inside nanotube bundles. The corrugation felt by the individual atom is so strong that the effective mass enhancement is very large: $m^{*} \simeq 18 m$ [44]. There is also a weaker opposing effect: $28 \%$ reduction in the well depth of the pair interaction mediated by the carbon environment [35]. Combining these effects and assuming the interaction range does not change significantly, we find that De Boer's number will decrease by a factor of 3.6 away from its purely one-dimensional value thus implying a liquid ground state. Similar outcome is expected for ${ }^{3} \mathrm{He}$.

\section{Molecular hydrogen}

The pair interaction between two hydrogen (or deuterium) molecules is commonly described by the semiempirical Silvera-Goldman potential [21]. Fig.1 shows this potential together with its Morse fit; the calculated Morse parameters are quoted in Table I.

As can be deduced from Figs. 5 and 6 , and Table I the many-body system of $H_{2}$ molecules is a Luttinger liquid with strongest effects of zero-point motion. It is characterized by $-5.14 K$ cohesive energy (ground-state energy per particle) which is an $84 \%$ reduction in magnitude away from the depth of the $\mathrm{H}_{2}-\mathrm{H}_{2}$ potential, largest Debye temperature of $121 K$, largest Luttinger liquid exponent $g \simeq 0.1$, largest quantum expansion of $0.82 \AA$, and largest, just under $18 \%$, relative fluctuation of the bond length.

The equilibrium distance between the $H_{2}$ molecules is $4.26 \AA$. As can be seen from Fig. 1 in a range around the equilibrium bond length significantly exceeding its rms fluctuation the Morse potential is a very good approximation to the Silvera-Goldman potential. The fit worsens at interparticle separations exceeding $5 \stackrel{\circ}{A}$; there are also deviations from the Silvera-Goldman potential at distances smaller than $3 \stackrel{\circ}{A}$. However at these compressions Hemley and collaborators [45] have found a softening effect unaccounted for by the Sivera-Goldman potential. We verified that the Morse potential shown in Fig.1 provides a very good fit to the Hemley-corrected version of the Silvera-Goldman potential.

Previous work [17] finds a liquid ground-state with the energy per particle to be $-4.8 K$ and the bond length of $4.6 \AA$. These values are close to our results. However we disagree with the existence of a liquid-solid transition found in Ref. [17] at higher density; such a transition is forbidden in one dimension. 
The building blocks of one-dimensional molecular hydrogen are $H_{2}$ molecules whose size of $0.75 \AA$ [37] is significantly smaller than the computed intermolecular distance of $4.26 \AA$. Such structure can be understood qualitatively from a complementary viewpoint:

There is exactly one electron per every hydrogen atom, and if the protons are arranged equidistantly, then the valence band is half-full, and the resulting system is an alkali metal [1]. However Peierls [46] noticed that in one dimension the energy can be further lowered by displacing every second nucleus by a prescribed distance. As a result of the period doubling the valence band becomes full, and the resulting dimer chain is an insulator. We conclude that one-dimensional molecular hydrogen is an example of Peierls-distorted one-dimensional structure; the dimers are hydrogen molecules.

Peierls' arguments rely on an adiabatic approximation which ignores zero-point motion of the nuclei; the former may change the answer qualitatively. Our theory which starts from interacting $H_{2}$ molecules shows that even for one-dimensional hydrogen the ground-state is a Peierlsdistorted insulator despite strong quantum fluctuations.

This conclusion may change upon application of pressure which brings hydrogen molecules closer to each other and increases relative fluctuation of the bond length. We argue that the distortion disappears and thus an insulator-metal transition takes place when all the hydrogen atoms become translationally identical. This transition is a one-dimensional version of the metallization transition predicted by Wigner and Huntington [37]. In one dimension the mechanism of the transition consists in "undoing" the Peierls distortion.

Since the bond between the two hydrogen molecules is significantly softer than that holding the $H_{2}$ molecule together, we assume that upon application of pressure, only the former decreases. Thus the translational equivalence of all the hydrogen atoms will be achieved when intermolecular spacing reaches the value of order $1.5 \AA$, twice the size of the $H_{2}$ molecule. With zero-pressure intermolecular spacing being $4.26 \AA$, this corresponds to compression by a factor of 2.84. The corresponding reduced pressure $p^{*} \gg 1$ can be found by inverting Eq.(48):

$$
p^{*} \simeq 2 e^{2\left\{Q_{0}-Q+\left[\sqrt{2} \lambda_{0} \ln \left(1+\frac{\pi}{2}\right)-\frac{1}{2}\right] e^{Q-Q_{0}}\right\}}
$$

Substituting here $Q_{0}=4.986, Q=H / l=2.174$, and $\lambda_{0}=0.2819$ we arrive at the reduced pressure of 545 . Our theory which does not explicitly consider electronic degrees of freedom fails in the vicinity of the inverse Peierls transition.

For molecular hydrogen in one dimension the unit of pressure is a force of $\epsilon / l \simeq 6.44 * 10^{-12} N$ strong. Multiplying this by 545 we find that $3.51 * 10^{-9} N$ force compressing one-dimensional hydrogen may suffice to induce a transition into metallic state. If this force is applied at the $1 \AA^{2}$ area, then the corresponding three- dimensional pressure will be $351 G P a$. We note that three-dimensional solid hydrogen subject to pressure that big still resists metallization [47]. Unfortunately the accuracy of our estimate is not great because of the exponential dependencies in (49) - the actual one-dimensional transition may happen at lower or larger pressures.

For hydrogen confined inside interstitial channels of carbon nanotube bundles the carbon environment effectively reduces the well depth of the pair interaction by $54 \%$ [35]. This effect alone would suffice to turn the many-body system of hydrogen molecules into a gas of $\left(\mathrm{H}_{2}\right)_{2}$ complexes. However if the effective mass enhancement is comparable to that for $\mathrm{He}$ [44], the liquid ground state might be restored.

As can be seen from Fig.6 and Table I, the ground state of molecular deuterium is a Luttinger liquid. The quantitative difference from the properties of molecular hydrogen is solely due to the fact that $D_{2}$ has a larger mass. The cohesive energy of the one-dimensional $D_{2}$ liquid is $-12.1 \mathrm{~K}$ (62\% reduction in the magnitude of the $D_{2}-D_{2}$ pair potential well), the Debye temperature is $99.8 \mathrm{~K}$ while the equilibrium distance between $D_{2}$ molecules is $3.94 \AA$.

\section{Heavier substances}

While discussing the physics of molecular hydrogen in one dimension we came to the conclusion that it can be viewed as an example of Peierls-distorted structure. The same arguments are applicable to any element with odd number of electrons: the period doubling should take place and the resulting system must be an insulator. We note that three-dimensional counterparts of these substances are metals. These observations imply that our theory is also applicable to substances which traditionally are not considered to belong to the molecular group. For example, one-dimensional lithium must be an insulating Luttinger liquid of $\mathrm{Li}_{2}$ molecules [48]. Similar to molecular hydrogen, under pressure it should undergo a metallization transition. Had we known the pair interaction between two $\mathrm{Li}_{2}$ molecules, we could have computed the properties of the lithium liquid. From the viewpoint of the quantum theorem of corresponding states molecular lithium is expected to occupy a place somewhere between $D_{2}$ and $\mathrm{Ne}$.

Table I also contains the Morse data for $N e$ and $A r$. They were obtained from the parameters of the LennardJones interaction potential [1] which is commonly used to describe these noble gases. Some of the properties of these substances in one dimension can be found in Table I and in Figs.5 and 6. The equilibrium interparticle spacing, cohesive energy and Debye temperature can be extracted from what is shown in the same manner as was done for lighter elements. As the underlying particles become heavier, the effect of zero-point motion decreases. 
For elements heavier than Ar quantum fluctuations can be ignored for most practical purposes.

\section{ACKNOWLEDGMENTS}

We thank M. Fowler, I. Harrison R. Kalas and T. J. Newman for valuable discussions.

This work was supported by the Thomas F. Jeffress and Kate Miller Jeffress Memorial Trust, and by the Chemical Sciences, Geosciences and Biosciences Division, Office of Basic Energy Sciences, Office of Science, U. S. Department of Energy.

* mail address: ek6n@virginia.edu

$\dagger$ mail address: xq8z@virginia.edu

\# mail address: mat3q@virginia.edu

[1] N. W. Ashcroft and N. D. Mermin, Solid State Physics (Saunders College Publishing, 1976).

[2] J. De Boer, Physica 14, 139 (1948); J. De Boer and B. S. Blaisse, Physica 14, 149(1948); J. de Boer and R. J. Lunbeck, Physica 14, 520 (1948).

[3] P. W. Anderson and R. G. Palmer, Nat. Phys. Sci. 231, 145 (1971); Phys. Rev. D 9, 3281 (1974); see also P. W. Anderson, Basic Notions of Condensed Matter Physics (Addison-Wesley Reading, Massachusetts, 1997), Section IIID.

[4] J. W Clark and N. C. Chao, Nat. Phys. Sci. 236, 37 (1972).

[5] N. D. Mermin and H. Wagner, Phys. Rev. Lett. 27, 1133 (1966), N. D. Mermin, J. Math. Phys. 8, 1061 (1967); P. C. Hohenberg, Phys. Rev. 158, 383 (1967).

[6] V. N. Popov, Teor. Mat. Fiz. 11, 354 (1972) [Theor. Math. Phys. 11, 65 (1972)]; K.B. Efetov and A. I. Larkin, Zh. Ekps. Teor. Fiz. 69, 764 (1975) [Sov. Phys. JETP 43, 390 (1976); F. D. M. Haldane, Phys. Rev. Lett. 45,1358 (1980); 47,1840 (1981); J. Phys. C 14, 2585 (1981); Phys. Lett. 81A, 153 (1981); E. B. Kolomeisky and J.P. Straley, Rev. Mod. Phys. 68, 175 (1996), and references therein. The harmonic liquid is a possible ground state if interparticle interaction is not more long-ranged than an inverse-square potential - this comes from the scaling behavior of the kinetic energy operator in the many-body Schrödinger equation.

[7] L. H. Nosanow, L. J. Parish, and F. J. Pinski, Phys. Rev. B 11, 191 (1975).

[8] S. Iijima, Nature (London), 354, 56 (1991).

[9] P. M.Ajayan and T. W. Ebbesen, Rep. Prog. Phys. 60, 1025 (1997).

[10] W. Teizer, R. B. Hallock, E. Dujardin, and T. W. Ebbesen, Phys. Rev. Lett. 82, 5305 (1999), and references therein.

[11] C. Dillon, K. M. Jones, T. A. Bekkedahl, C. H. Kiang, D. S. Bethune, and M. J. Heben, Nature (London), 386,
377 (1997); F. Darkrim and D. Levesque, J. Chem. Phys. 109, 4981 (1998); J. M. Ogden, Physics Today 55, 69 (2002), and references therein.

[12] F. Schreck, L. Khaykovich, K. L. Corwin, G. Ferrari, T. Bourdel, J. Cubizolles, and C. Salomon, Phys. Rev. Lett. 87, 080403 (2001); A. Görlitz, J. M. Vogels, A. E. Leanhardt, C. Raman, T. L. Gustavson, J. R. Abo-Shaeer, A. P. Chikkatur, S. Gupta, S. Inouye, T. Rosenband, and W. Ketterle, Phys. Rev. Lett 87, 130402 (2001).

[13] K. E. Strecker, G. B. Partridge, A. G. Truscott, and R. G. Hulet, Nature (London) 417, 150 (2002); L. Khaikovich, F. Schreck, G. Ferrari, T. Bourdel, J. Cubizolles, L. D. Carr, Y. Castin, C. Salomon, Science 296, 1290 (2002).

[14] M. A. Kasevich, C. R. Acad. Sci. IV 2, 497 (2001).

[15] G. Stan, V. H. Crespi, M. W. Cole, and M.Boninsegni, J. Low Temp. Phys. 113,447 (1998); M. Boninsegni and S. Moroni, J. Low Temp. Phys. 118, 1 (2000).

[16] M. C. Gordillo, J. Boronat, and J. Casulleras, Phys. Rev. B 61, R878 (2000).

[17] M. C. Gordillo, J. Boronat,and J. Casulleras, Phys. Rev. Lett. 85, 2348 (2000).

[18] E. Krotscheck, M. D. Miller, and J. Wojdylo, Phys. Rev. B 60, 13028 (1999), E. Krotscheck, M. D. Miller, ibid. 60, 13038 (1999).

[19] A. I. Karaevskii and V. V. Lubashenko, Phys. Rev. B 60, 12091 (1999).

[20] P. M. Morse, Phys. Rev. 34; L. D. Landau and E. M. Lifshitz, Quantum Mechanics (Pergmon, New York, 1977), Problem 4 to Section 23.

[21] I. F. Silvera and V. V. Goldman, J. Chem. Phys. 69, 4209 (1978), I. F. Silvera, Rev. Mod. Phys. 52, 393 (1980).

[22] J. B. Kogut, Rev. Mod. Phys. 51, 659 (1979), and references therein.

[23] E. B. Kolomeisky and J.P. Straley, Phys. Rev. B 62, 301 (2000).

[24] A. O. Caldeira and A. J. Leggett, Phys. Rev. Lett. 46, 211 (1981)

[25] The first term of (13) written in terms of the Fourier transform $h(\omega)$ is identical to the first term of Eq.(5) in [23].

[26] R. P. Feynman, Statistical Mechanics (Benjamin, Boston, 1972), p. 67.

[27] Y. Saito, Z. Phys. B 32, 75 (1978).

[28] J. D. Weeks, Phys. Rev. B 26, 3998 (1982); in Phase Transformations in Solids, edited by T. Tsakalakos (Elsevier, New York, 1984), p. 597.

[29] R. Lipowsky, D. M. Kroll, and R. K. P. Zia, Phys. Rev. B 27, 4499 (1983).

[30] M. P. A. Fisher and W. Zwerger, Phys. Rev. B 32, 6190 (1985), and references therein.

[31] E. B. Kolomeisky, R. M. Konik, and X. Qi, Phys. Rev. B 66, 075318 (2002).

[32] H. Kleinert, Path Integrals in Quantum Mechanics, Statistics, and Polymer Physics (World Scientific, Singapore, 1995), Chapter 5 and references therein.

[33] E. Brézin, B. I. Halperin, and S. Leibler, Phys. Rev. Lett. 50, 1387 (1983).

[34] F. Lindemann, Phys. Z. (Leipzig), 11, 69 (1910).

[35] M. K. Kostov, M. W. Cole, J. C. Lewis, P. Diep, and J. K. Johnson, Chem Phys. Lett. 332, 26 (2000); M. K. Kostov, J. C. Lewis, and M. W. Cole, cond-mat/0010015. 
[36] M. Boninsegni, S. -Y. Lee, and V. H. Crespi, Phys. Rev. Lett. 86, 3360 (2001), and references therein.

[37] E. Wigner and H. B. Huntington, J. Chem. Phys. 3, 764 (1935), and references therein.

[38] M. M. Calbi, M. W. Cole, S. M. Gatica, M. J. Bojan, and G. Stan, Rev. Mod. Phys. 73, 857 (2001), and references therein.

[39] W. Kolos and L. Wolniewicz, J. Chem. Phys. 43, 2429 (1965).

[40] R. D. Etters, J. V. Dugan, and R. W. Palmer, J. Chem. Phys. 62, 313 (1975).

[41] R. Aziz, V. P. S. Nain, J. S. Carley, W. L. Taylor and G. T. McConville, J. Chem. Phys. 70, 4330 (1979).

[42] R. E. Grisenti, W. Schöllkopf, J. P. Toennies, G. C. Hegerfeldt, T. Köhler, and M. Stoll, Phys. Rev. Lett. 85, 2284 (2000), and references therein.

[43] E. P. Bashkin, Zh. Eksp. Teor. Fiz. 78, 360 (1980) [Sov. Phys. JETP 51, 181 (1980)].

[44] M. W. Cole, V. H. Crespi, G. Stan, C. Ebner, J. M. Hartman, and M. Boninsegni, Phys. Rev. Lett. 84, 3883 (2000), and references therein.

[45] R. J. Hemley, H. K. Mao, L. W. Finger, A. P. Jephcoat, R. M. Hazen, and C. S. Zha, Phys. Rev. B 42, 6458 (1990).

[46] R. E. Peierls, Quantum Theory of Solids (Oxford, 1955), Sesction 5.3; More Surprises in Theoretical Physics (Princeton University Press, 1991), Section 2.3.

[47] P. Loubeyre, F. Occelli, and R. LeToullec, Nature (London) 416, 613 (2002), and references therein.

[48] It has been demonstrated in J. B. Neaton and N. W. Ashcroft, Nature (London) 400, 141 (1999) that under high pressure similar pairing into $L i_{2}$ molecules takes place in ordinary solid lithium. 